\title{
TRPM7 mediates kidney injury, endothelial hyperpermeability and mortality during endotoxemia
}

\author{
Sebastian Gatica ${ }^{1,2}$. Vicente Villegas ${ }^{1}$ Alejandro Vallejos $\mathbb{1}^{1,2} \cdot$ Pedro Olivares $^{1}$ - Víctor Aballai ${ }^{3,4}$. \\ Felipe Lagos-Meza ${ }^{3,4}$. Cesar Echeverria ${ }^{5}$ Claudio Cabello-Verrugio ${ }^{1,2,6} \cdot$ Diego Varela $^{3,4} \cdot$ Felipe Simon $\mathbb{D}^{1,2}$
}

Received: 15 January 2019 / Revised: 24 June 2019 / Accepted: 19 July 2019 / Published online: 23 August 2019

(c) United States \& Canadian Academy of Pathology 2019

\begin{abstract}
Sepsis is the main cause of mortality in patients admitted to intensive care units. During sepsis, endothelial permeability is severely augmented, contributing to renal dysfunction and patient mortality. $\mathrm{Ca}^{2+}$ influx and the subsequent increase in intracellular $\left[\mathrm{Ca}^{2+}\right]_{\mathrm{i}}$ in endothelial cells (ECs) are key steps in the establishment of endothelial hyperpermeability. Transient receptor potential melastatin 7 (TRPM7) ion channels are permeable to $\mathrm{Ca}^{2+}$ and are expressed in a broad range of cell types and tissues, including ECs and kidneys. However, the role of TRPM7 on endothelial hyperpermeability during sepsis has remained elusive. Therefore, we investigated the participation of TRPM7 in renal vascular hyperpermeability, renal dysfunction, and enhanced mortality induced by endotoxemia. Our results showed that endotoxin increases endothelial hyperpermeability and $\mathrm{Ca}^{2+}$ overload through the TLR4/NOX-2/ROS/NF-kB pathway. Moreover, endotoxin exposure was shown to downregulate the expression of VE-cadherin, compromising monolayer integrity and enhancing vascular hyperpermeability. Notably, endotoxin-induced endothelial hyperpermeability was substantially inhibited by pharmacological inhibition and specific suppression of TRPM7 expression. The endotoxin was shown to upregulate the expression of TRPM7 via the TLR4/NOX-2/ROS/NF-kB pathway and induce a TRPM7-dependent EC Ca ${ }^{2+}$ overload. Remarkably, in vivo experiments performed in endotoxemic animals showed that pharmacological inhibition and specific suppression of TRPM7 expression inhibits renal vascular hyperpermeability, prevents kidney dysfunction, and improves survival in endotoxemic animals. Therefore, our results showed that TRPM7 mediates endotoxemia-induced endothelial hyperpermeability, renal dysfunction, and enhanced mortality, revealing a novel molecular target for treating renal vascular hyperpermeability and kidney dysfunction during endotoxemia, sepsis, and other inflammatory diseases.
\end{abstract}

These authors contributed equally: Sebastian Gatica, Vicente Villegas

Supplementary information The online version of this article (https:// doi.org/10.1038/s41374-019-0304-z) contains supplementary material, which is available to authorized users.

Felipe Simon

fsimon@unab.cl

1 Facultad de Ciencias de la Vida, Universidad Andres Bello, Republica 239, 8370146 Santiago, Chile

2 Millennium Institute on Immunology and Immunotherapy, Alameda 340, 8331150 Santiago, Chile

3 Programa de Fisiología y Biofísica, Instituto de Ciencias Biomédicas, Facultad de Medicina, Universidad de Chile,

\section{Introduction}

Sepsis is the major cause of mortality in critically ill patients admitted to intensive care units. Sepsis is a multifaceted host response characterized by severe systemic inflammation [1, 2]. Patients undergoing sepsis progress to multiple organ dysfunction syndrome (MODS), exhibiting mortality rates up to $60 \%[3,4]$. In particular, acute kidney dysfunction occurs in $\sim 50 \%$ of

8380453 Santiago, Chile

4 Millennium Nucleus of Ion Channels-Associated Diseases (MiNICAD), Universidad de Chile, Santiago, Chile

5 Facultad de Medicina, Universidad de Atacama, Copayapu 485, 1531772 Copiapo, Chile

6 Center for the Development of Nanoscience and Nanotechnology (CEDENNA), Universidad de Santiago de Chile, Santiago, Chile 
hospitalized septic patients in the USA [5], up to half of whom require dialysis [6]. Unfortunately, current treatments remain unsatisfactory [7, 8]. Sepsis is frequently induced and reinforced by the accumulation of Gramnegative bacterial endotoxin lipopolysaccharide (LPS) in the bloodstream, a condition known as endotoxemia [9-11].

Detrimental actions of sepsis and endotoxemia progress as a consequence of an uncontrolled systemic activation of endothelial cells (ECs) and the immune system, involving the secretion of proinflammatory cytokines and generation of $\mathrm{NAD}(\mathrm{P}) \mathrm{H}$ oxidase-dependent oxidative stress [10-13]. ECs are susceptible to damage induced by circulating pathogens, endotoxins, proinflammatory cytokines, and reactive oxygen and nitrogen species, which may lead to endothelial dysfunction if the damage is sustained [14-17]. Endothelial dysfunction promotes critical alterations, including vascular leakage and hypoperfusion associated with sepsis-induced MODS [18-22]. Specifically, in the kidney, macro- and microcirculatory disturbances, oxidative stress, and the surge of inflammatory mediators via NF- $\mathrm{KB}$ activation contribute to acute kidney dysfunction in septic patients [23].

Endothelial barrier function relies on cell-to-cell physical associations maintained by endothelial adhesion proteins (EAPs, e.g., VE-cadherin and PECAM-1 / CD-31) that regulate the transport of molecules through the monolayer [24-26]. Exposure of ECs to endotoxins, pro-inflammatory cytokines, or oxidative stress induces a downregulation in the expression of EAPs [27-31], which compromises barrier integrity. This phenomenon is accompanied by an increase in fibroblast-like characteristics and has been described as endothelial-tomesenchymal transition [17, 27, 32, 33]. During sepsis, endothelial permeability drastically increases and is speculated to contribute significantly to the pathogenesis of sepsis-induced kidney injury. In fact, leakage of plasma from the vascular space and increased interstitial edema contribute to the compression of peritubular capillaries, compromising blood flow. Thus, subjecting parenchymal areas to limited oxygen supply and ischemic damage induces the generation of oxidative stress and inflammation, which further compromises renal function [34-36] and patient likelihood for recovery $[15,37,38]$.

It has been reported that $\mathrm{Ca}^{2+}$ influx, and the subsequent increase in intracellular $\mathrm{Ca}^{2+}$ concentration $\left(\left[\mathrm{Ca}^{2+}\right]_{\mathrm{i}}\right)$ in ECs, is a required step for endothelial hyperpermeability. Accordingly, inhibition of $\mathrm{Ca}^{2+}$ influx significantly decreases endothelial hyperpermeability [39-41], even in the presence of major downregulators of EAP, such as proinflammatory cytokines or oxidative stress [41, 42].
Transient receptor potential melastatin 7 (TRPM7) ion channels are permeable to divalent cations $\mathrm{Ca}^{2+}$ and $\mathrm{Mg}^{2+}$ and are ubiquitously expressed in a broad range of cell and tissue types, including ECs and kidneys [43-46]. Interestingly, TRPM7 also carries a cytoplasmic kinase domain with enzymatic activity, for which it is referred to as a chanzyme. Although its intracellular presence has been extensively reported [47, 48], its participation in biological processes has been less frequently described [49]. Nevertheless, conciliation of function between its channel and enzyme remains a matter of study [47]. The role of TRPM7 in the pathogenesis of renal vascular hyperpermeability or sepsis-induced kidney injury has not yet been studied. Related evidence has indicated that TRPM7 is overexpressed in the kidney in ischemia-reperfusion models in vivo [50-52]. Moreover, intrarenal delivery of shRNA against TRPM7 prevents apoptosis, expression of fibrotic transformation marker $\alpha$ SMA, and tissue fibrosis in a mouse model of renal transplantation [53]. Furthermore, TRPM7 is associated with inflammation, apoptosis, tubulointerstitial damage, and decreased renal function, and silencing of TRPM7 improves injury parameters, renal histology, and function [54]. Therefore, this study aimed to elucidate the participation of TRPM7 in endothelial hyperpermeability induced by endotoxemia focusing on kidney dysfunction.

Our results showed that endotoxin increases endothelial hyperpermeability and $\left[\mathrm{Ca}^{2+}\right]_{\mathrm{i}}$ through the TLR4/NOX-2/ROS/ NF-kB pathway. In addition, endotoxin was shown to downregulate VE-cadherin expression, compromising monolayer integrity and enhancing hyperpermeability. Moreover, TRPM7 expression was shown to be induced by endotoxemia, which is mediated through the TLR4/NOX-2/ROS/NF- $\mathrm{KB}$ pathway. Interestingly, specific suppression and pharmacological inhibition of TRPM7 inhibited endothelial hyperpermeability and attenuated $\mathrm{Ca}^{2+}$ overload in ECs exposed to endotoxin. Remarkably, in vivo experiments performed in endotoxemic animals showed that specific expression suppression and pharmacological inhibition of TRPM7 inhibited renal vascular hyperpermeability, preventing kidney dysfunction, and improved survival by $20-40 \%$ in endotoxemic animals.

Overall, our results contribute to the understanding of the molecular bases of endotoxemia-induced vascular hyperpermeability, revealing a new molecular target with substantial potential for hyperpermeability during endotoxemia, sepsis, and other inflammatory diseases.

\section{Materials and methods}

\section{Animals and experimental groups}

Male Sprague-Dawley rats, weighing $100-120 \mathrm{~g}$ and $\sim 4-5$ weeks old were housed in individual cages and given $a d$ 
libitum access to food and water. Four experimental protocols, assessing (A) vascular permeability, (B) renal function, (C) survival, and (D) inflammation, were approved by the Commission of Bioethics and Biosafety from Universidad Andres Bello. Rats were separated into four groups with concurrent experimental conditions for the four protocols:

Group 1: Carvacrol vehicle- or adenovirus (AdV) vehicletreated sham endotoxemia group. Rats were subjected to two $24 \mathrm{~h}$ spaced injections of carvacrol vehicle (i.p.) or $\mathrm{AdV}$ vehicle (i.v.) in $300 \mu \mathrm{L}$ boluses. After $30 \mathrm{~min}$, rats were injected i.p. with $300 \mu \mathrm{L}$ sterile saline solution $(n=6$ for each protocol, except for protocol C, for which $n=10$ ).

Group 2: Carvacrol- or $\mathrm{AdV}_{\text {shTRPM7-treated sham endo- }}$ toxemia group. Rats were subjected to two $24 \mathrm{~h}$ spaced injections of carvacrol $\left(40 \mathrm{mg} / \mathrm{kg}\right.$, i.p.) or $\operatorname{AdV}_{\text {shTRPM7 }}(3 \times$ $10^{10}$ viral particles, i.v.) in $300 \mu \mathrm{L}$ boluses. After $30 \mathrm{~min}$, rats were injected i.p. with $300 \mu \mathrm{L}$ sterile saline solution. ( $n=6$ for each protocol, except for protocol C, for which $n=10$ ).

Group 3: Carvacrol vehicle- or AdV vehicle-treated endotoxemic group. The rats were subjected to two $24 \mathrm{~h}$ spaced injections of carvacrol vehicle (i.p.) or AdV vehicle (i.v.) in $300 \mu \mathrm{L}$ boluses. After $30 \mathrm{~min}$, rats were injected with LPS $(20 \mathrm{mg} / \mathrm{kg}$, i.p.). ( $n=6$ for each protocol, except for protocol C, for which $n=10$ ).

Group 4: Carvacrol- or $\mathrm{AdV}_{\text {shTRPM7 }}$-treated endotoxemic group. The rats were subjected to two $24 \mathrm{~h}$ spaced injections of carvacrol $(40 \mathrm{mg} / \mathrm{kg}$, i.p. $)$ or $\mathrm{AdV}_{\text {shTRPM7 }}\left(3 \times 10^{10}\right.$ viral particles, i.v.) in $300 \mu \mathrm{L}$ boluses. After $30 \mathrm{~min}$, rats were injected with LPS ( $20 \mathrm{mg} / \mathrm{kg}$, i.p.). ( $n=6$ for each protocol, except for protocol C, for which $n=10)$.

For vascular permeability analyses (experimental protocol A), $24 \mathrm{~h}$ after induction of endotoxemia, or sham endotoxemia, rats were subjected to the in vivo permeability assay (detailed below).

For renal function analyses (experimental protocol B), $24 \mathrm{~h}$ after induction of endotoxemia, or sham endotoxemia, rats were anesthetized with isoflurane and whole blood was collected via i.c. puncture.

For survival observations (experimental protocol C), 12, 24, 36, 48, 60, and $72 \mathrm{~h}$ after induction of endotoxemia, or sham endotoxemia, rats were inspected visually. After $72 \mathrm{~h}$, surviving animals were euthanized by isoflurane overdose.

For inflammation analyses (experimental protocol D), $24 \mathrm{~h}$ after induction of endotoxemia, or sham endotoxemia, rats were anesthetized with isoflurane, whole blood was collected via i.c. puncture, and kidneys were harvested for histologic observation.

\section{AdV production and infection}

For complementary alignment, the primers (FWR: 5'-T CGAGGCACCTTTATATCATTATTCAAGAGATAATG ATATAAAGGTGCCTTTTT-3'
REV: 5'-GAAAAAGGCACCTTTATATCATTATCTC TTGAATAATGATATAAAGGTGCC-3') were diluted to $100 \mu \mathrm{M}$ in a buffer solution containing (in $\mathrm{mM}$ ) $50 \mathrm{NaCl}, 10$ Tris- $\mathrm{HCl}, 10 \mathrm{MgCl}_{2}$ and $10 \mu \mathrm{g} / \mathrm{ml} \mathrm{BSA,} \mathrm{pH} 7.9$ and incubated at $95{ }^{\circ} \mathrm{C}$ for $5 \mathrm{~min}$ followed by $3 \mathrm{~h}$ incubation at $25^{\circ} \mathrm{C}$. The shRNA was then subcloned into the pShuttleU6 vector (a kind gift from Ronald Kahn, Addgene plasmid \# 13428) using XbaI and SalI (New England Biolabs). The adenoviral vectors were generated using the AdEasy system as described previously [55]. Briefly, homologous recombination was carried out by electroporation of BJ5183 cells (Agilent Technologies) with PmeI linearized DNA (pAD/RFP AdV) was used as control. Recombinant adenoviral plasmids were digested with PacI (New England Biolabs) and transfected into AdHEK293 cells with Lipofectamine 2000 (Life Technologies) according to the manufacturer's guidelines. Following observation of cytopathic effects (CPEs; 14-21 days), the cells were scraped and subjected to four freeze-thaw cycles in a dry-ice methanol bath. The resulting supernatant was used to infect a $10 \mathrm{~cm}$ dish of $70 \%$ confluent AdHEK293 cells. Following observation of CPEs after 5-7 days, viral particles were purified and expanded by infecting four plates of AdHEK293 cells. For in vitro infection: the $\mathrm{AdV}$ dose contained $1 \times 10^{8}$ viral particles added to cultured ECs (for in vitro specificity and efficiency experiments see Supplementary Fig. S1). For in vivo infection, $A d V_{\text {shTRPM7 }}$ was administered i.v. The AdV dose contained $3 \times 10^{10}$ viral particles injected in a $300 \mu \mathrm{l}$ volume. (For in vivo specificity and efficiency experiments see Supplementary Fig. S2.)

\section{EC line culture}

HUVEC-derived EC line EA.hy926 was obtained from ATCC (CRL-2922, Manassas, VA) and cultured at $37^{\circ} \mathrm{C}$ in a 5\%:95\% $\mathrm{CO}_{2}$ :air atmosphere in DMEM-low glucose (GIBCO, USA) supplemented with 10\% FBS, $2 \mathrm{mM}$ glutamine, and $50 \mathrm{U} / \mathrm{ml}$ penicillin-streptomycin (SigmaAldrich, St. Louis, USA). Experiments were performed in serum-reduced media (1\% FBS) and cells were cultured in $1 \%$ FBS $24 \mathrm{~h}$ before experiments.

\section{Small interfering RNAs (siRNAs) and transfections}

SiGENOME SMARTpool siRNA (four separated siRNAs) against TRPM7, TLR4, NOX-1, NOX-2, and NOX-4, and a control siRNA (nontargeting siRNA) was purchased from Dharmacon (Dharmacon, Lafayette, CO). In brief, cells were plated overnight in 24-well plates and then transfected with $5 \mathrm{nmol} / \mathrm{L}$ siRNA using DharmaFECT 4 transfection reagent (Dharmacon) according to manufacturer guidelines in serum-free 
medium for $6 \mathrm{~h}$. Experiments were performed $24-48 \mathrm{~h}$ after transfection.

\section{In vitro permeability assay}

ECs were cultured to full confluency for $24 \mathrm{~h}$ on gelatincoated Boyden chamber mounted inside the wells of 24-well plates (Transwells, Costar Transwell, $0.4 \mu \mathrm{m}$ pore size, 12-mm diameter, Corning Inc., New York, USA). In indicated experiments confluent monolayers were treated for $1 \mathrm{~h}$ with CLI-095 $(10 \mu \mathrm{M})$, DPI $(5 \mu \mathrm{M})$, Apo $(10 \mathrm{mM})$, NAC $(5 \mathrm{mM})$, GSH $(1 \mathrm{mM})$, SC3060 $(5 \mu \mathrm{M})$, carvacrol $(100 \mu \mathrm{M}), 2$-APB $(10-150 \mu \mathrm{M}), \mathrm{Gd}^{3+}(10 \mu \mathrm{M})$, and $\mathrm{Zn}^{2+}$ $(10 \mu \mathrm{M})$. Then, without interrupting treatment, indicated experiments were added endotoxin $(0,10,20,35$, and $50 \mu \mathrm{g} / \mathrm{ml} \mathrm{LPS}$ ) for 3 days. Then, FITC-dextran $40 \mathrm{kDa}$ $(0.5 \mathrm{mg} / \mathrm{ml}$, Sigma-Aldrich, St. Louis, USA) was added to the chamber. After $90 \mathrm{~min}$ of incubation, a $50 \mu \mathrm{l}$ sample was taken from the plate well and fluorescence was measured using a fluorescence plate reader (excitation $485 \mathrm{~nm}$, emission $520 \mathrm{~nm}$ ). Alternatively, cells were transiently transfected with specific siRNA or nontargeting siRNA in separate plates (for efficiency and specificity experiments see Fig. 3a and Supplementary Fig. S1). Twelve hours post transfection, cells were cultured to full confluency on gelatin-coated transwells for $24 \mathrm{~h}$. Then, in indicated experiments, confluent monolayers were added endotoxin, and permeability was measured as described above. Results are expressed as normalized permeability compared with control group.

\section{In vivo permeability assay}

Rats from experimental protocol A were anesthetized with isoflurane and injected with Evans blue dye (EBD, $80 \mathrm{mg} /$ $\mathrm{kg}, i . v$.) for $10 \mathrm{~min}$. EBD binds to circulating plasma proteins and extravasates to tissue parenchima at sites of increased vascular permeability. Then, rats were euthanized and perfused with saline solution via the left ventricle to wash excess intravascular dye. Inner abdominal wall was removed and washed in cold saline solution and immediately documented using a wide zoom stereo microscope (Olympus SZX16). Left kidney was removed, washed in cold saline solution, snap-frozen using liquid nitrogen vapor phase, and stored at $-80{ }^{\circ} \mathrm{C}$ until histological processing. Right kidney was removed and washed in cold saline solution. Both, right kidney and inner abdominal wall, were gently dried using a paper towel, weighted, and prepared for EBD extraction following a modification of protocols described elsewhere [56-58]. Briefly, inner abdominal wall and right kidney were weighted and homogenized using a tissue homogenizer (OMNI international, Kennesaw, GA, USA) with $100 \%$ trichloroacetic acid in a $1: 1$ or
1:2 (mg:ml) proportion, respectively. Homogenates were centrifuged at $4180 \times g$ for $36 \mathrm{~min}$ and supernatant optical density was determined spectrophotometrically at $630 \mathrm{~nm}$ using microplate reader ELx800 (BioTek Instruments Winooski, VT, USA).

\section{Plasma measurements of kidney markers dysfunction}

Blood collected from animals from experimental protocol B was immediately centrifuged at $4000 \mathrm{rpm}$ for $10 \mathrm{~min}$ at $4{ }^{\circ} \mathrm{C}$ to separate the plasma, which was then used to measure kidney dysfunction. Plasma levels of creatinine (CRE) and blood urea nitrogen (BUN) were measured using the Piccolo Xpress Chemistry Analyzer, General Chemistry, 13 panel (Abaxis, CA, USA), according to the manufacturers' instructions.

\section{Measurement of circulating cytokines}

Blood collected from animals from experimental protocol D was immediately centrifuged at $4,000 \mathrm{rpm}$ for $10 \mathrm{~min}$ at $4^{\circ}$ $\mathrm{C}$ to separate the plasma, which was then used to measure cytokines. TNF- $\alpha$, IL-1 $\beta$, and IL- 6 were measured with an enzyme-linked immunosorbent assay (ELISA) according to instructions by the manufacturer (R\&D Systems Inc., MN, USA).

\section{Western blot}

Whole-cell proteins from cells treated with endotoxin $(20$ $\mu \mathrm{g} / \mathrm{ml}$ ) or vehicle were extracted in cold lysis buffer. Supernatants were collected and stored in the same lysis buffer. Protein extracts and supernatants were subjected to SDS-PAGE, and resolved proteins were transferred to a nitrocellulose or PVDF membrane. Blocked membranes were incubated with primary antibody against VE-cadherin, TRPM7, or tubulin, washed twice, and incubated with corresponding HRP-conjugated secondary IgG antibody. For a detailed list of antibodies used in western blot experiments see Supplementary Table S1. Immunoreactive bands were detected using enhanced chemiluminescence (Bio-Rad, CA) and images were acquired using Fotodyne FOTO/Analyst Luminary Workstations Systems (Fotodyne, Inc., Hartland, WI). Protein content was determined by densitometric scanning of immunoreactive bands, and intensity values were obtained by densitometry of individual bands normalized against tubulin.

\section{Measurement of $\left[\mathrm{Ca}^{2+}\right]_{\mathrm{i}}$}

After treatments or transfections, ECs were washed with Hank's solution, and then incubated for $30 \mathrm{~min}$ at $37^{\circ} \mathrm{C}$ in 
the dark in the presence of $3 \mu \mathrm{M}$ Fura-2-acetoxymethyl ester (Fura-2AM; Molecular Probes). Loaded cells were then washed twice with Hank's solution and placed in a perfusion chamber on the stage of an inverted microscope (Olympus IX-81, UPLFLN 40XO $40 \times / 1.3$ oil-immersion objective). $\mathrm{Ca}^{2+}$ concentration was determined by measuring Fura-2AM emission after excitation at 380 and $340 \mathrm{~nm}$. Measured ratios were calibrated against standard solutions (Invitrogen) of known $\mathrm{Ca}^{2+}$ concentration as described previously [59-61].

\section{Preparation for histological observation}

Right kidneys from animals from experimental protocol A were embedded in O.C.T. compound (Tissue-Tek, Sakura Finetek, CA) and $60 \mu \mathrm{m}$ sections were obtained using a microtome-cryostat. Sections were mounted on positively charged slides and documented at low magnification using a wide zoom stereo microscope (Olympus SZX16).

Both kidneys from animals from experimental protocol D were fixed in PBS-buffered $4 \%$ formalin, washed, dehydrated in ethanol, cleared in xylene, embedded in paraffin, and $4 \mu \mathrm{m}$ sections were obtained using a rotary microtome. Sections were mounted on positively charged slides, rehydrated, stained using Harris' hematoxylin and 5\% eosin Y, dehydrated, mounted using a hydrophobic mounting medium, inspected using an upright light microscope, and documented using a mounted USB camera (Moticam 2300, Motic).

\section{Reagents and inhibitors}

The following reagents and inhibitors were used: LPS from Escherichia coli (O127:B8, Sigma-Aldrich, St. Louis, USA), TLR4 inhibitor (CLI-095, InvivoGen, San Diego, CA), Fluo-4 (Invitrogen), FITC-dextran (TdB Consultancy, Uppsala, Sweden), NF-кB inhibitor SC3060 (Santa Cruz Biotechnology), NAD(P)H oxidase inhibitors diphenyleneiodonium (DPI, Sigma-Aldrich, St. Louis, USA) and apocynin (Apo, Sigma-Aldrich), cell permeable antioxidant N-Acetylcysteine (NAC, Tocris), reduced form of glutathione (GSH, $1 \mathrm{mM}$, Sigma-Aldrich), and TRP channel blockers carvacrol (Sigma) and 2-APB (Calbiochem, CA, USA). Buffers and salts were purchased from Merck Biosciences.

\section{Data analysis}

All results are presented as mean $\pm \mathrm{SD}$. Statistical differences were assessed using one-way analysis of variance (ANOVA) (or nonparametric Kruskal-Wallis) followed by Dunn's post hoc test or two-way ANOVA followed by Tukey's post hoc test. Differences were considered significant at $p<0.05$. See the figure legends for specific test used. Plasma measurements were performed by one-way ANOVA (Kruskall-Wallis) followed by Dunn's post test. Log-rank tests were used to determine survival rates.

\section{Results}

\section{Endotoxin induces endothelial hyperpermeability through a mechanism mediated by the TLR4/NOX/ ROS/NF-KB pathway}

First, we investigated whether endotoxin can induce endothelial hyperpermeability. Therefore, EC monolayers were exposed to endotoxin $(0-50 \mu \mathrm{g} / \mathrm{ml})$, and permeability was analyzed at $24 \mathrm{~h}$. The endotoxin dose range did not change cell viability (Supplementary Fig. S3). The endotoxin promoted a dose-dependent increase in the permeability of the monolayer (Fig. 1a) and a dose-dependent decrease in VEcadherin expression level in ECs that constituted the monolayer (Fig. 1b).

To determine whether endotoxin elicits its action through TLR4, we performed a sequence of experiments to test how specific TLR4 inhibitor CLI-095 was. Use of CLI-095 significantly prevents hyperpermeability upon exposure to the endotoxin (Fig. 1c). Use of a blocking antibody against TLR4 prior to incubation with endotoxin prevented endothelial hyperpermeability as well (Supplementary Fig. S4a). Transfection of ECs with a siRNA against TLR4 (siTLR4 downregulation efficiency $>90 \%$, Supplementary Fig. S4b) prevented hyperpermeability as significantly as CLI-095 (Supplementary Fig. S4c). These results, in accordance with reports published previously [62-64], strongly suggest that CLI-095 significantly hinders endotoxin signaling by specifically inhibiting TLR4. This receptor exerts its action through the NOX/ROS signaling pathway. Therefore, we incubated ECs with the NOX inhibitors DPI and apocynin and then exposed ECs to the endotoxin. Both DPI (Fig. 1d) and apocynin (Fig. 1e) effectively prevented hyperpermeability upon exposure to the endotoxin.

To determine the specific participation of endothelial NOX isoforms, we performed experiments using siRNA against isoforms NOX-1, NOX-2, and NOX-4 [12, 65, 66]. The results showed that EC transfection with siRNA against NOX-2 prevented hyperpermeability upon exposure to the endotoxin (Fig. 1g). In contrast, EC transfection with siRNAs against NOX-1 (Fig. 1f) and NOX-4 (Fig. 1h) failed to prevent hyperpermeability upon exposure to the endotoxin. Therefore, NOX-2, but not NOX-1 or NOX-4, participates in endotoxininduced endothelial hyperpermeability.

To evaluate the participation of oxidative stress, we exposed EC monolayers to the antioxidant agent NAC and the reducing molecule GSH. Both molecules significantly prevented 
A

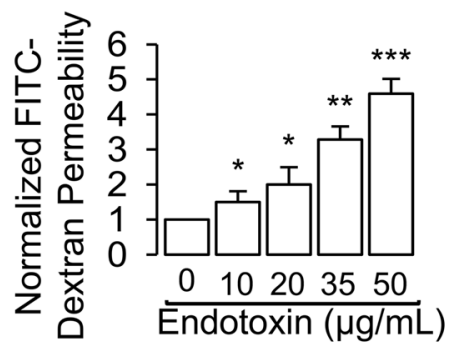

B

VE-Cad $=-\cdots$

Tubulin $=-\cdots$

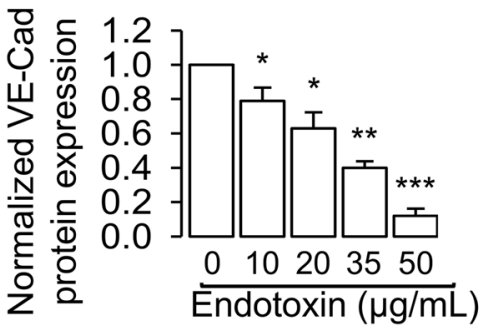

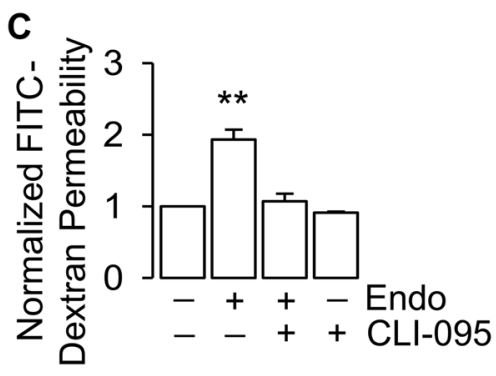
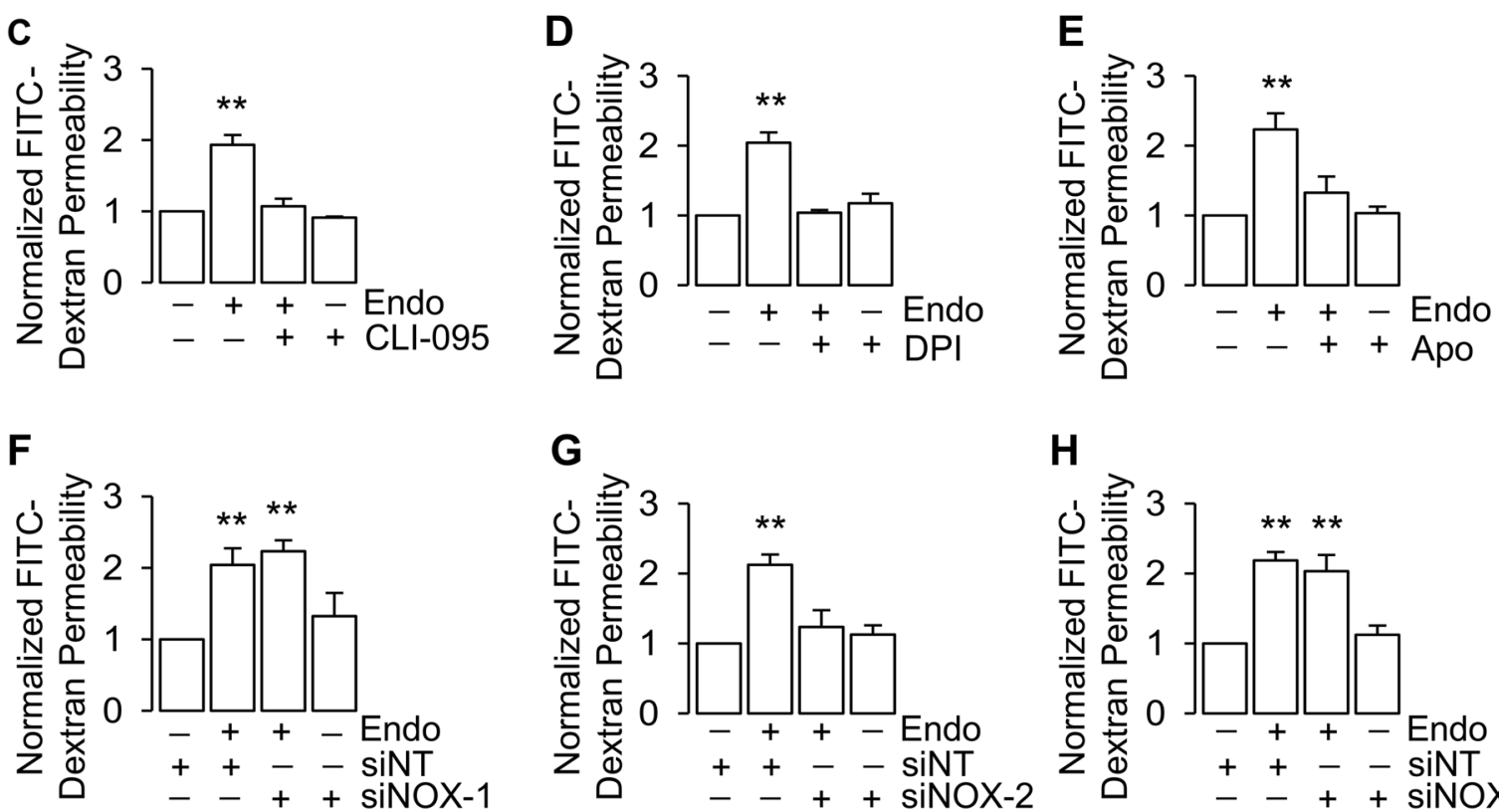

G

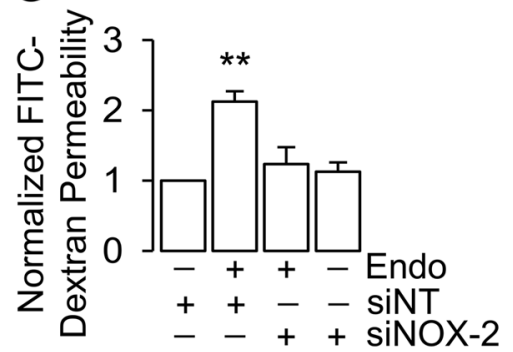

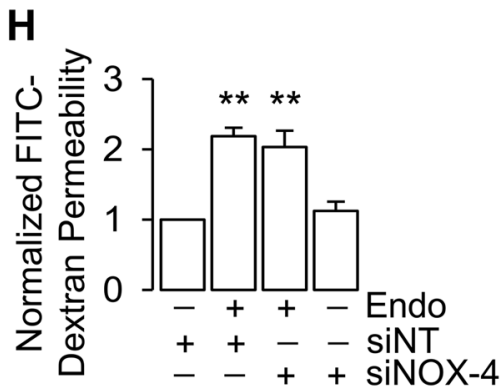

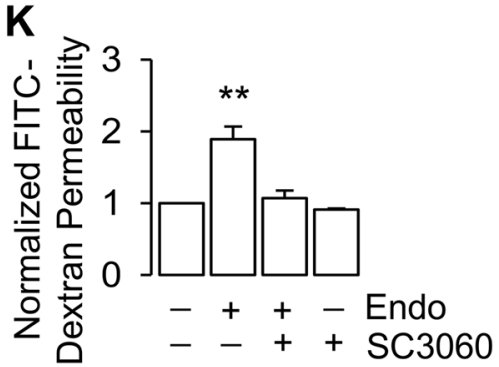

Fig. 1 Endotoxin induces endothelial hyperpermeability through a mechanism mediated by TLR4/NOX/ROS/NF- $\mathrm{BB}$ pathway. a Endotoxin increases EC monolayer permeability. ECs were exposed to endotoxin $(0,10,20,35$, and $50 \mu \mathrm{g} / \mathrm{ml})$ for $24 \mathrm{~h}$ and endothelial permeability to FITC-dextran was analyzed. $(N=8)$. b Endotoxin induces a decrease in VE-cadherin expression. ECs were exposed to endotoxin $(0,10,20,35$, and $50 \mu \mathrm{g} / \mathrm{ml})$ for $24 \mathrm{~h}$ and VE-cadherin protein expression was analyzed. Protein levels were normalized against tubulin. Data are expressed relative to control $(N=5)$. $\mathbf{c}-\mathbf{k}$ Endotoxin induces endothelial hyperpermeability through the TLR4/NOX/ROS/ $\mathrm{NF}-\kappa \mathrm{B}$ pathway. ECs were preincubated for $1 \mathrm{~h}$ in the presence or

hyperpermeability upon exposure to the endotoxin (Fig. 1i, j). Moreover, both molecules significantly prevented hyperpermeability induced by $\mathrm{H}_{2} \mathrm{O}_{2}$ (Supplementary Fig. S5). absence of TLR4 inhibitor CLI-095 (c), NOX inhibitors DPI (d) and apocynin (e), antioxidant agent NAC (i), reducing molecule GSH (j), or NF-kB inhibitor SC3060 (preincubated for $6 \mathrm{~h}$ ) (k). A different set of ECs were transfected using siRNAs against NOX isoforms NOX-1 (f), NOX-2 (g), and NOX-4 (h). Cells were then exposed to endotoxin $(20 \mu \mathrm{g} / \mathrm{ml})$ for $24 \mathrm{~h}$, after which, endothelial permeability of FITCdextran was analyzed. $(N=4-9)$. Results are expressed as normalized permeability compared with control. Statistical differences were assessed by one-way analysis of variance (ANOVA) (Kruskal-Wallis) followed by Dunn's post hoc test. $* p<0.05, * * p<0.01, * * * p<0.001$ against control condition. Histogram bars show mean \pm SD

TLR4 signaling regulates protein expression by activa-

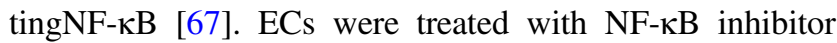
SC3060 and then exposed to the endotoxin. Treatment with 
Fig. 2 Endotoxin-induced endothelial hyperpermeability is reduced by TRPM7 ion channel inhibitors. ECs were preincubated for $1 \mathrm{~h}$ in the presence or absence of TRPM7 inhibitors carvacrol (a), 2-APB (b), $\mathrm{Gd}^{3+}(\mathbf{c})$, and $\mathrm{Zn}^{2+}(\mathbf{d})$, and exposed to endotoxin $(20 \mu \mathrm{g} / \mathrm{ml})$ for $24 \mathrm{~h}$, after which, endothelial permeability to FITC-dextran was analyzed. Results are expressed as normalized permeability compared with control. EC $\left[\mathrm{Ca}^{2+}\right]_{\mathrm{i}}$

measurements in the presence or absence of TRPM7 inhibitors carvacrol, 2-APB (e), $\mathrm{Gd}^{3+}$, and $\mathrm{Zn}^{2+}$ (f); TLR4 and NOX inhibitors CLI-095, DPI, apocynin (g), NOX isoforms suppressors siNOX1, siNOX2, and siNOX4 (h), NAC, GSH (i); or NF-kB inhibitor SC3060 (j) under endotoxic or control conditions. $\mathrm{Ca}^{2+}$ concentrations were determined by measuring Fura-2 emission after excitation at 380 and $340 \mathrm{~nm}$. Measured ratios were calibrated against standard solutions of known $\mathrm{Ca}^{2+}$ concentration. Statistical differences were assessed by one-way analysis of variance (ANOVA) (Kruskal-Wallis) followed by Dunn's post hoc test. $* * p<0.01$, against control condition. Histogram bars show mean $\pm \mathrm{SD}(N=4-8)$
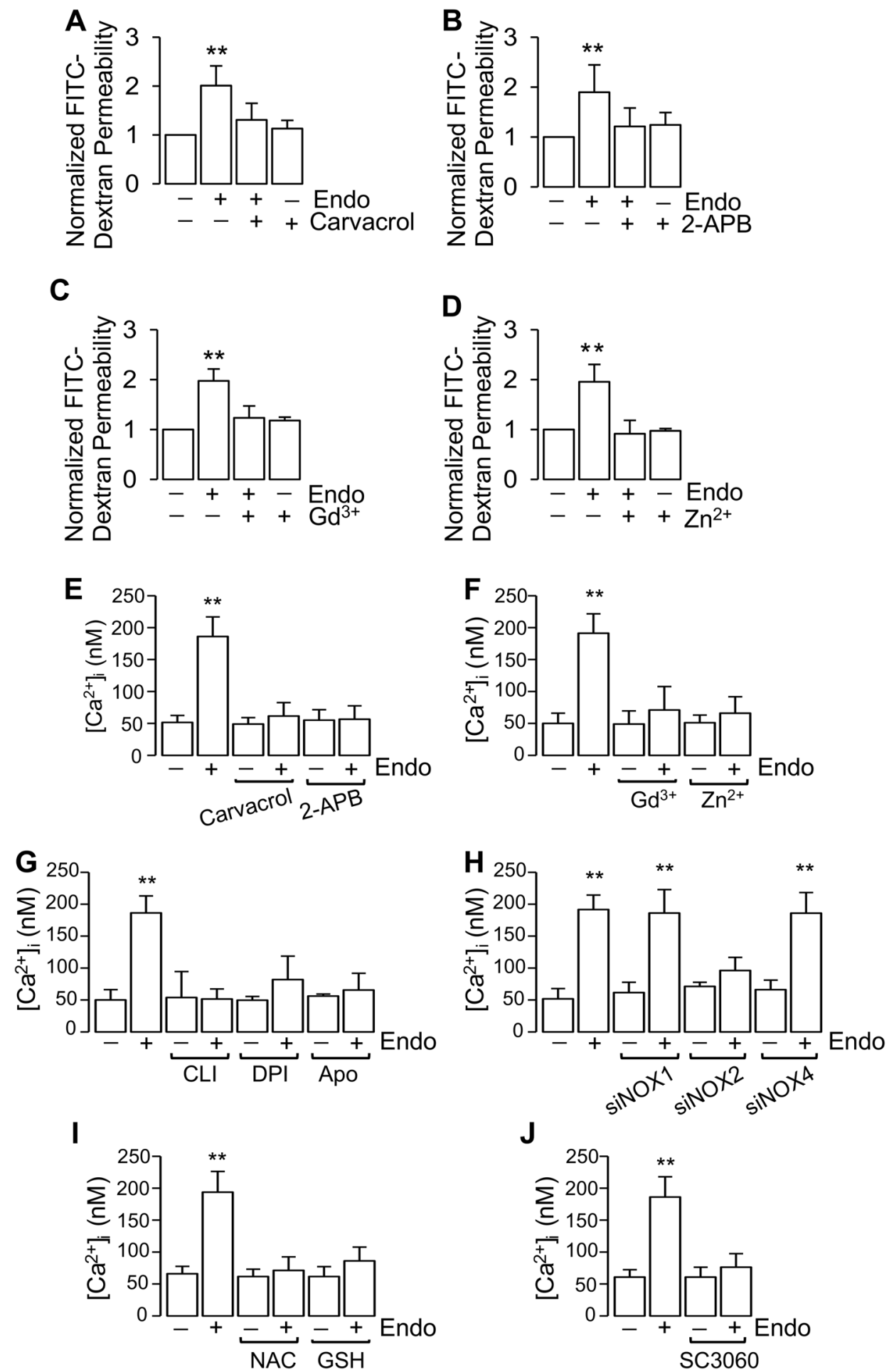

SC3060 significantly prevented hyperpermeability upon exposure to the endotoxin (Fig. 1k). Therefore, these results suggest that the TLR4/NOX-2/ROS/NF- $\mathrm{kB}$ intracellular signaling pathway mediates endotoxin-induced endothelial hyperpermeability.

\section{TRPM7 mediates endotoxin-induced endothelial hyperpermeability}

We investigated the participation of TRPM7 in endotoxininduced endothelial hyperpermeability by analyzing the 


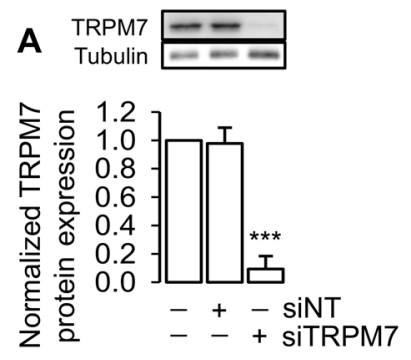

E
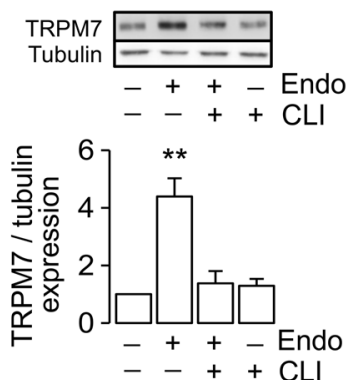

B

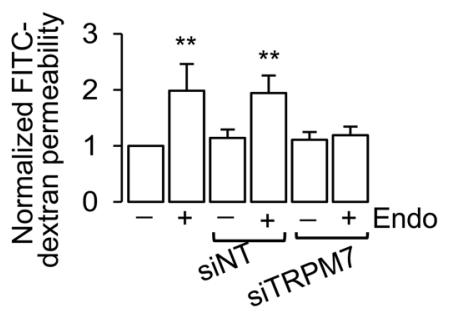

$\mathbf{F}$
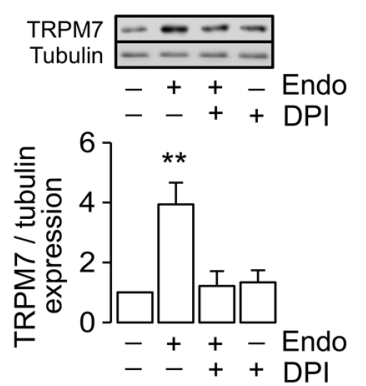

Fig. 3 TRPM7 mediates endotoxin-induced endothelial hyperpermeability. a Downregulation of TRPM7 expression by siRNA. ECs were transfected with specific siRNA against human TRPM7 (siTRPM7) or nontargeting siRNA (siNT). b TRPM7 mediates endotoxin-induced endothelial hyperpermeability. ECs transfected with siTRPM7 or siNT, or nontransfected cells, were exposed to endotoxin $(20 \mu \mathrm{g} / \mathrm{ml})$ for $24 \mathrm{~h}$ and endothelial permeability to FITC-dextran was analyzed. c Endotoxin increases TRPM7 protein expression. ECs were exposed to endotoxin $(0,10$, and $20 \mu \mathrm{g} / \mathrm{ml})$ for $24 \mathrm{~h}$ and TRPM7 protein expression was analyzed. d siTRPM7 or siNT, or nontransfected EC $\left[\mathrm{Ca}^{2+}\right]_{\mathrm{i}}$ measurements under endotoxic or control conditions. Endotoxin

effect of the TRPM7 inhibitors carvacrol [68-70] and 2APB [71, 72]. Treatment of EC monolayers with carvacrol (Fig. 2a) or 2-APB (Fig. 2b) prevented hyperpermeability upon exposure to the endotoxin. Similar results were observed using the nonspecific TRPM7 inhibitors $\mathrm{Gd}^{3+}$ (Fig. 2c) and $\mathrm{Zn}^{2+}$ (Fig. 2d) [44, 73, 74].

As we have reported previously [33], endotoxin induces a 2.5 -fold increase in $\left[\mathrm{Ca}^{2+}\right]_{\mathrm{i}}$ in ECs (Supplementary Fig. S6). Thus, we investigated whether TRPM7 inhibitors were able to inhibit the endotoxin-induced $\left[\mathrm{Ca}^{2+}\right]_{\mathrm{i}}$ increase by loading attached ECs with calcium-sensitive fluorescent indicator Fura-2AM. Carvacrol and 2-APB (Fig. 2e) significantly prevented the increase in $\left[\mathrm{Ca}^{2+}\right]_{i}$ induced after exposure to the endotoxin. A similar effect was observed when the cells were treated with $\mathrm{Gd}^{3+}$ and $\mathrm{Zn}^{2+}$ (Fig. 2f). Interestingly, the TLR4 inhibitor CLI-095 and the NOX inhibitors DPI and apocynin were all effective in preventing the increase in $\left[\mathrm{Ca}^{2+}\right]_{\mathrm{i}}$ induced after exposure to the endotoxin (Fig. 2g). In addition, NOX-2, but not NOX-1 or NOX-4, mediated the increase in $\left[\mathrm{Ca}^{2+}\right]_{\mathrm{i}}$ induced after exposure to the endotoxin (Fig. 2h). Similarly, NAC and GSH also prevented the increase in $\left[\mathrm{Ca}^{2+}\right]_{\mathrm{i}}$ induced after

C TRPM7 $=$

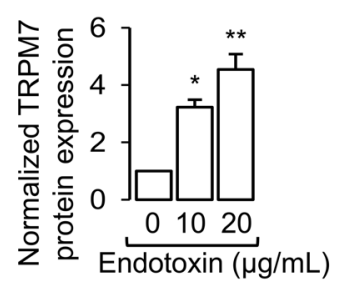

G
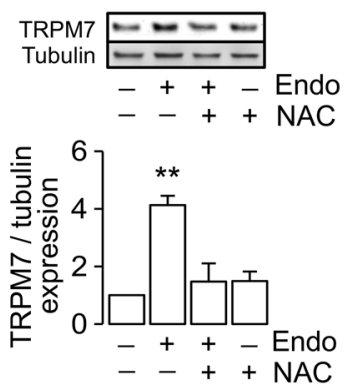

D

H
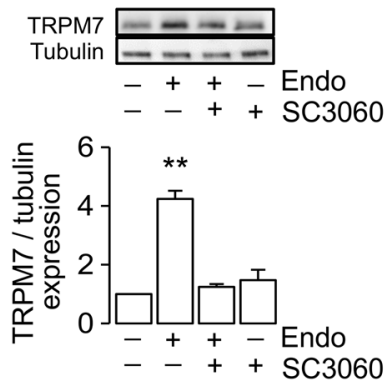

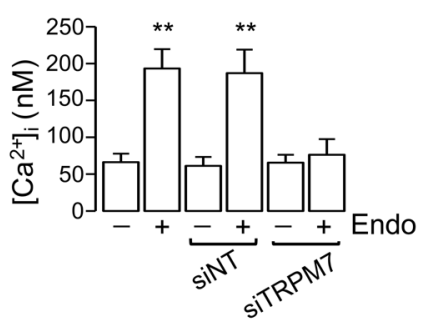

increases TRPM7 protein expression through TLR4/NOX/ROS/NF$\kappa B$ pathway. ECs were preincubated for $1 \mathrm{~h}$ in the presence or absence TLR4 inhibitor CLI-095 (e), NOX inhibitor DPI (f), antioxidant agent NAC (g), or NF-kB inhibitor SC3060 (h), then exposed to endotoxin $(20 \mu \mathrm{g} / \mathrm{ml})$ for $24 \mathrm{~h}$, and processed for TRPM7 protein expression analysis. Protein levels were normalized against tubulin, and the data are expressed relative to nontransfected cells. Statistical differences were assessed by one-way analysis of variance (ANOVA) (Kruskal-Wallis) followed by Dunn's post hoc test. ${ }^{*} p<0.05,{ }^{* *} p<$ $0.01, * * * p<0.001$ against control condition. Histogram bars show mean $\pm \mathrm{SD}(N=3-6)$

exposure to the endotoxin (Fig. 2i). In addition, the NF-кB inhibitor SC3060 prevented the increase in $\left[\mathrm{Ca}^{2+}\right]_{\mathrm{i}}$ induced after exposure to the endotoxin (Fig. 2j). These results suggest that TRPM7 activity mediates the increase in $\left[\mathrm{Ca}^{2+}\right]$ ${ }_{i}$ induced by the endotoxin, which is mediated by the TLR4/ NOX-2/ROS/NF- $\mathrm{kB}$ signaling pathway.

Next, we applied an experimental strategy using molecular biology approaches to downregulate TRPM7 expression and investigated whether this ion channel is involved in endotoxin-induced endothelial hyperpermeability. We used a specific siRNA targeting the human isoform of TRPM7 (siTRPM7). The siRNA downregulation efficiency was $>90 \%$ compared with ECs transfected with a nontargeting siRNA sequence used as control (siNT) (Fig. 3a). Furthermore, siTRPM7 did not affect the expression of the TRPM7 homologues TRPM2 and TRPM6 (Supplementary Fig. S7). After exposure to the endotoxin, siNT-transfected EC monolayers showed similar permeability characteristics to nontransfected monolayers (Fig. 3b). Notably, siTRPM7 transfection prevented endothelial hyperpermeability upon exposure to the endotoxin, indicating that TRPM7 is a key factor that mediates endothelial permeability (Fig. 3b). 
We previously reported that the TRPM7 expression level increases upon endotoxin challenge in hippocampal neurons [73]. Our results showed that TRPM7 expression level also increased in ECs when they were exposed to the endotoxin (Fig. 3c). Interestingly, TRPM7 suppression significantly attenuated the increase in $\left[\mathrm{Ca}^{2+}\right]_{\mathrm{i}}$ induced by the endotoxin (Fig. 3d). Next, we investigated whether the TLR4/NOX/ $\mathrm{ROS} / \mathrm{NF}-\kappa \mathrm{B}$ intracellular signaling pathway is involved in the increased expression level of TRPM7 induced by the endotoxin. Treatment of ECs with CLI-095 (Fig. 3e), DPI (Fig. 3f), NAC (Fig. 3g), and SC3060 (Fig. 3h) prevented the increase in TRPM7 expression levels induced by endotoxin. Therefore, these results suggest that the TLR4/ $\mathrm{NOX} / \mathrm{ROS} / \mathrm{NF}-\kappa \mathrm{B}$ intracellular signaling pathway mediates the increase in TRPM7 expression levels induced by endotoxin.

\section{TRPM7 activity inhibition and suppression of TRPM7 expression decrease renal vascular hyperpermeability during endotoxemia}

Endotoxin administration was effective in inducing changes that were concordant with the accepted criteria for the diagnosis of severe sepsis in humans $[75,76]$. To demonstrate this, we recorded systolic blood pressure $\left(\mathrm{P}_{\mathrm{S}}\right)$ and heart rate $\left(\mathrm{f}_{\mathrm{H}}\right)$ of the rats 90 min after i.p. saline or endotoxin challenge. Rats that were subjected to endotoxemia exhibited a significant decrease in systolic blood pressure (hypotension) and tachycardia compared with rats that were injected with saline solution, indicating that the LPS treatment generated hemodynamic alterations concordant with severe sepsis (Supplementary Table S2).

To evaluate TRPM7 channel participation in renal vascular permeability during endotoxemia, endotoxemic rats were pretreated with TRPM7 pharmacological inhibitors. Kidneys from rats treated with carvacrol (Fig. 4a, b) or 2APB (Supplementary Fig. S8) were significantly resistant to vascular hyperpermeability after $24 \mathrm{~h}$ of endotoxemia. Interstitial accumulation of EBD was significantly increased in extracts from endotoxemic rats compared with extracts from vehicle-treated rats (Fig. 4a, b). Interestingly, the accumulation of EBD showed no significant difference in endotoxemic rats treated with carvacrol (Fig. 4a, b) or 2APB (Supplementary Fig. S8) alone.

To directly analyze the role of TRPM7 in regulating endothelial permeability during endotoxemia, we performed loss-of-function studies. Because TRPM7 knockout leads to early embryonic lethality [77], an AdV encoding a shRNA specific for TRPM7 ( $\left.\mathrm{AdV}_{\text {shTRPM7 }}\right)$ was constructed using a strategy similar to that reported in previous study conducted by Sun et al. [78].

First, we analyzed TRPM7 suppression by infecting cultured ECs with $\mathrm{AdV}_{\text {shTRPM7 }}$ and analyzing TRPM7 expression.
Infection with $\mathrm{AdV}_{\text {shTRPM7, }}$, but not with control $\mathrm{AdV}$ encoding for RFP $\left(\mathrm{AdV}_{\mathrm{RFP}}\right)$, downregulated TRPM7 expression with $>95 \%$ efficiency (Supplementary Fig. S1a, b). AdV $\mathrm{AshRPM}_{\text {shT }}$ infection did not affect the expression of the TRPM7 homologues TRPM2 and TRPM6 (Supplementary Fig. S1c). Then, rats were injected i.v. with $\mathrm{AdV}_{\text {shTRPM7 }}$ or $\mathrm{AdV}_{\mathrm{RFP}} 24 \mathrm{~h}$ prior to evaluation. We found that TRPM7 protein and mRNA expression were substantially downregulated in fresh protein extracts from mesenteric endothelial cells (RMECs) from

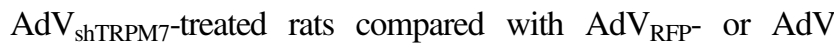
vehicle-treated rats (Supplementary Fig. S2a-c). Furthermore, immunocytochemical detection in whole intact aortas extracted from $\mathrm{AdV}_{\text {shTRPM7-}}$, $\mathrm{AdV}_{\mathrm{RFP}^{-}}$, or $\mathrm{AdV}$ vehicle-treated rats showed a significant decrease in the TRPM7 expression level (Supplementary Fig. S2d). Interfering RNAs may induce activation of innate response pathways leading to an upregulation of interferon (IFN) genes mediated by the dsRNA-dependent protein kinase PKR [79, 80]. Analysis of IFN- $\beta$ levels in plasma samples from $\mathrm{AdV}_{\text {shTRPM7 }}{ }^{-}, \mathrm{AdV}_{\mathrm{RFP}^{-}}$, and $\mathrm{AdV}$ vehicle-treated rats revealed no significant increase in IFN- $\beta$ (Supplementary Fig. S9a). Western blot analysis of RMECs from $\mathrm{AdV}_{\text {shTRPM7 }}{ }^{-}, \mathrm{AdV}_{\mathrm{RFP}^{-}}$, and $\mathrm{AdV}$ vehicle-treated rats revealed no significant increase in PKR expression levels (Supplementary Fig. S9b). Finally, we investigated whether TRPM7 downregulation affects rat survival. Survival of

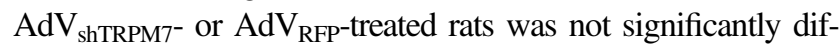
ferent from $\mathrm{AdV}$ vehicle-treated rats in the timeframe used in this study (not shown). Therefore, $\operatorname{AdV}_{\text {shTRPM7 }}$ infection efficiently and specifically downregulates TRPM7 without activating innate responses, inducing MODS, or affecting the survival of infected animals.

Then, inflammatory consequences of endotoxemia and differences after TRPM7 activity inhibition and suppression were assessed in blood and kidney tissues collected after $24 \mathrm{~h}$ of endotoxemia or sham endotoxemia with or without $\mathrm{AdV}_{\text {shTRPM7 }}$ or its corresponding vehicle. Biochemical quantification of circulating proinflammatory cytokines shows that levels of TNF $\alpha$, IL- $1 \beta$, and IL-6 are significantly higher in endotoxemia with respect to normal conditions, and such rise is significantly prevented for IL-6 under carvacrol or AdV $\mathrm{V}_{\text {shTRPM7 }}$ stimulation, and for TNF- $\alpha$ under $\mathrm{AdV}_{\text {shTRPM7 }}$ stimulation. Consistent with results shown in Supplementary Fig. S9, AdV $\mathrm{V}_{\text {shTRPM7 }}$ alone was not able to increase levels of TNF $\alpha$, IL-1 $\beta$, or IL-6, significantly, and neither was carvacrol alone (Supplementary Table S3). Microscopic examination of the cortex shows that normal (Veh/Sal, Carvacrol/Sal, and $\mathrm{AdV}_{\text {shTRPM7 }} / \mathrm{Sal}$ group) kidney histology is characterized by well-conserved spaces defined primarily by Bowman's spaces, proximal and distal tubules, and collection ducts. However, under endotoxemia (Veh/Endo group), these spaces drastically collapse configuring swollen filtration units with virtually no Bowman's space and very little 


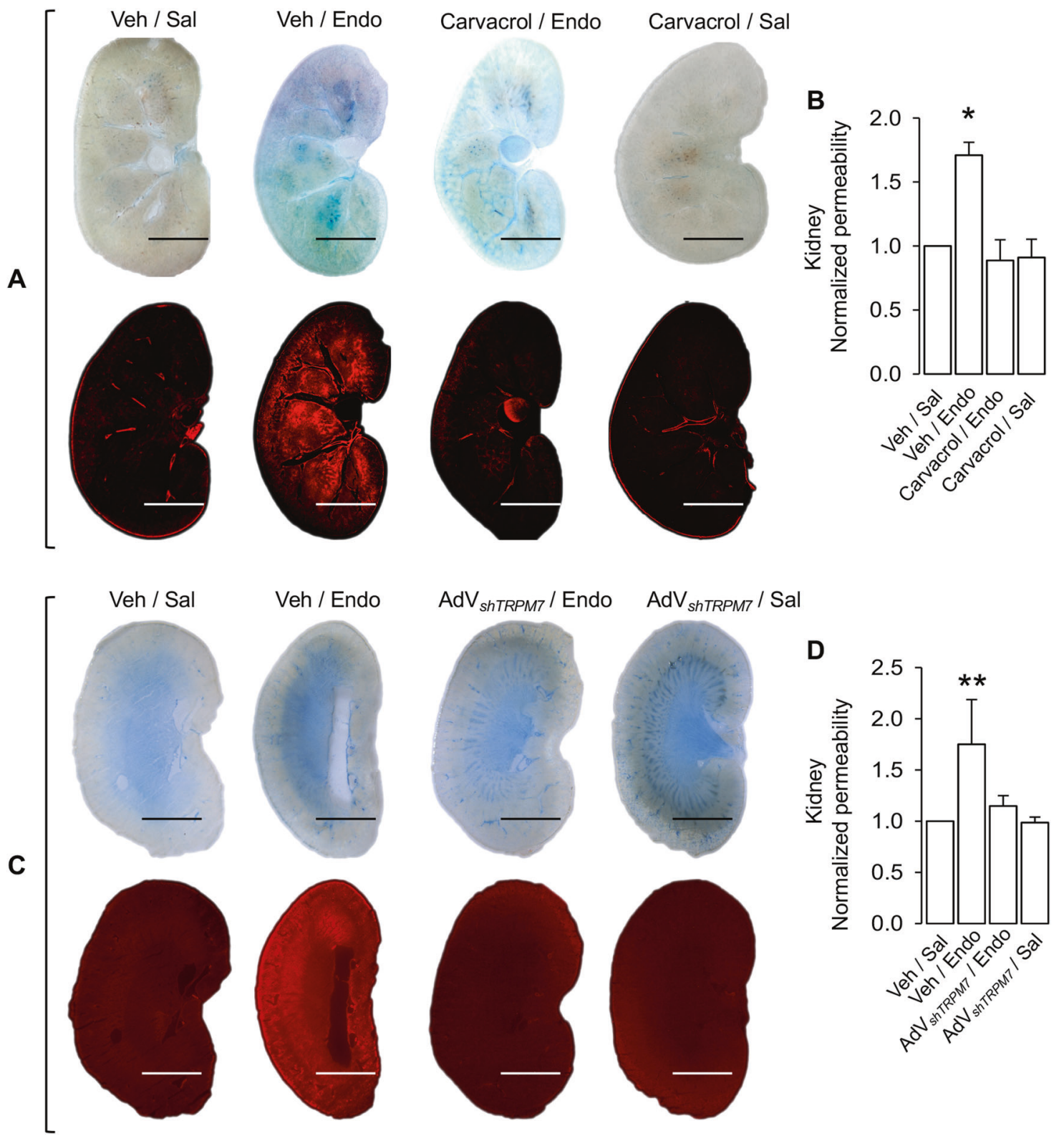

Fig. 4 TRPM7 activity inhibition and suppression of TRPM7 expression decrease endothelial hyperpermeability in kidneys during endotoxemia. Distribution of accumulated EBD throughout interstitial kidney territories from animals treated with carvacrol (a) or $\mathrm{AdV}_{\text {ShTRPM7 }}$ (c). Quantification of EBD extracted from whole kidneys from animals treated with carvacrol (b) or $\mathrm{AdV}_{\text {shTRPM7 }}(\mathbf{d})$. Animals were treated with $40 \mathrm{mg} / \mathrm{kg}$ carvacrol i.p., $\operatorname{AdV}_{\text {shTRPM7 }}$ i.v., or corresponding vehicle. Twenty-four hours later a reinforcing dose of carvacrol, $\mathrm{AdV}_{\mathrm{ShTRPM} 7}$, or vehicle, was administered. One hour later,

distinction of the tubular structures. This swollen picture is significantly reversed by treating animals with carvacrol or $\mathrm{AdV}_{\text {shTRPM7 }}$ (Supplementary Fig. S10). Interestingly, no morphologic impression of apoptosis or necrosis were found, and no significant differences in leukocyte infiltrates throughout the cortex could be observed for any of the experimental groups. animals were injected with $20 \mathrm{mg} / \mathrm{kg}$ LPS or saline solution i.p. to induce endotoxemia. Twenty-four hours later, rats were anesthetized and assayed for endothelial permeability in vivo. Kidneys were harvested and processed for EBD extraction. EBD content was normalized to total sample weight. Statistical differences were assessed by one-way analysis of variance (ANOVA) (Kruskal-Wallis) followed by Dunn's post hoc test. $* p<0.05$, against control condition. Histogram bars show mean $\pm \mathrm{SD}(N=6$ per group). Scale bar $=0.5 \mathrm{~cm}$. Image alterations: only background was removed

Next, we investigated the specific participation of TRPM7 in renal vascular permeability during endotoxemia. Rats were injected i.v. with $\mathrm{AdV}_{\text {shTRPM7 }}$ or the $\mathrm{AdV}$ vehicle. Twenty-four hours later, a second dose of $\mathrm{AdV}_{\text {shTRPM7 }}$ or the AdV vehicle was administered, and endotoxemia was induced by injecting $20 \mathrm{mg} / \mathrm{kg}$ LPS i.p. Twenty-four hours later, renal vascular permeability was analyzed. Kidneys 
from $\mathrm{AdV}_{\text {shTRPM7 }}$-treated rats were significantly resistant to vascular hyperpermeability after $24 \mathrm{~h}$ of endotoxemia (Fig. 4c, d). Interstitial accumulation of EBD was significantly increased in extracts from endotoxemic rats compared with extracts from AdV vehicle-treated rats (Fig. 4c, d). Remarkably, the accumulation of EBD showed no significant difference in endotoxemic rats infected with $\mathrm{AdV}_{\text {shTRPM7 }}$ (Fig. 4c, d). Similar results were observed in control rats that were only infected with $\mathrm{AdV}_{\text {shTRPM7 }}$.

\section{TRPM7 activity inhibition and suppression of TRPM7 expression protect against kidney dysfunction during endotoxemia}

During sepsis and endotoxemia kidney function is severely compromised [34-36]. Therefore, we evaluated the effect of carvacrol or $\mathrm{AdV}_{\text {ShTRPM7 }}$ on rat kidney function during endotoxemia. Blood analysis of rats treated with carvacrol (Fig. 5a, b) or injected with $\operatorname{AdV}_{\text {shTRPM7 }}$ (Fig. 5c, d) showed no significant changes in the levels of CRE and BUN after $24 \mathrm{~h}$ of endotoxemia, whereas changes in these markers of kidney dysfunction were significantly different in endotoxemic rats treated with the vehicle solutions. Interestingly, treatment with carvacrol or injection of $\mathrm{AdV}_{\text {shTRPM7 }}$ alone did not significantly affect the CRE or BUN levels (Fig. 5).

\section{TRPM7 activity inhibition and suppression of TRPM7 expression increase survival against endotoxemia}

To investigate the potentially beneficial effects of inhibiting or suppressing TRPM7, we analyzed the effects of carvacrol (Fig. 6a) or $\operatorname{AdV}_{\text {shTRPM7 }}$ (Fig. 6b) on endotoxemic animal survival over $72 \mathrm{~h}$. A deleterious effect of endotoxemia was observed with $80 \%$ lethality at $72 \mathrm{~h}$ (Fig. 6). Remarkably, inhibition of TRPM7 with carvacrol decreased endotoxemia lethality by $20 \%$ ( $60 \%$ survival; $p=0.0324$ compared with the vehicle-treated group, Fig. 6a), while suppression of TRPM7 with $\mathrm{AdV}_{\text {shTRPM7 }}$ decreased endotoxemia lethality by $40 \%$ ( $40 \%$ survival; $\mathrm{p}=0.0122$ compared with the AdV vehicletreated group, Fig. 6b).

\section{Discussion}

Endothelial hyperpermeability is a key phenomenon that predisposes septic patients to vascular leakage and edema. The accumulation of interstitial fluids impairs organ function by increasing interstitial pressure and compromising microvascular perfusion $[81,82]$. This leads to a decrease in effective volume and blood pressure, dramatically increasing the risk of death [83]. Therefore, endothelial hyperpermeability is not merely a side effect of sepsis, but rather a major contributor to its morbidity and mortality [84].
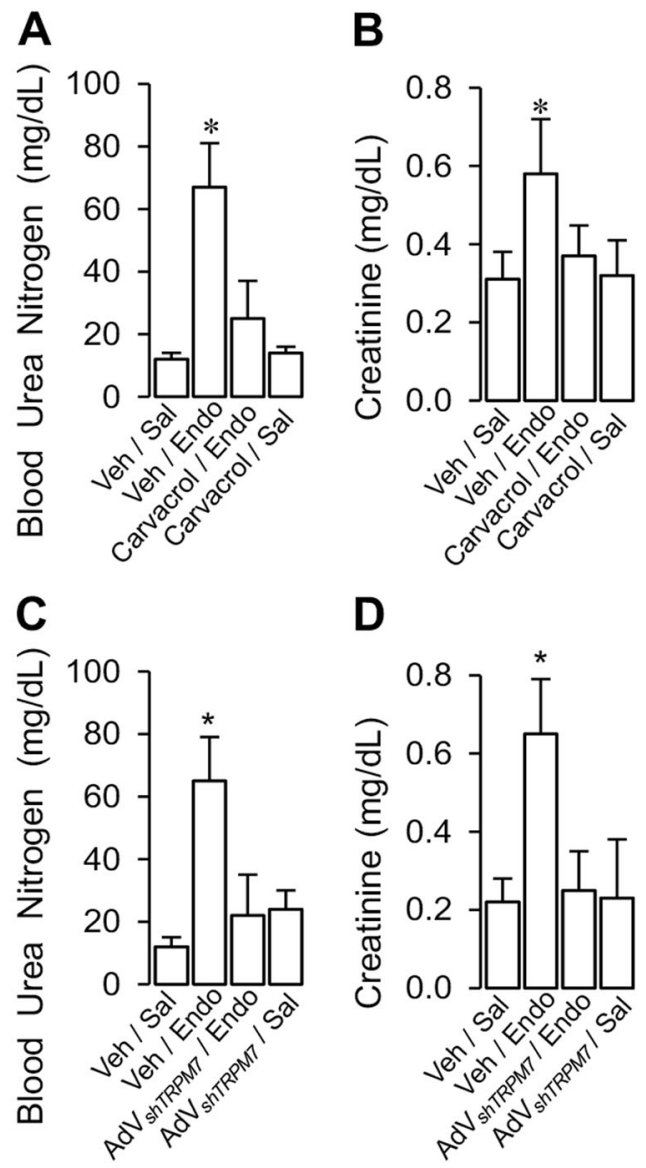

Fig. 5 TRPM7 activity inhibition and suppression of TRPM7 expression protect against kidney dysfunction during endotoxemia. Serum creatinine and blood urea nitrogen were measured in animals treated with carvacrol $(\mathbf{a}, \mathbf{b}), \operatorname{AdV}_{\text {shTRPM7 }}(\mathbf{c}, \mathbf{d})$, or corresponding vehicle. Statistical differences were assessed by two-way analysis of variance (ANOVA) followed by Bonferroni's post hoc test. $* p<0.05$, $* * * p<0.001$ against Veh/Sal condition. Histogram bars show mean \pm $\mathrm{SD}(N=6$ per group)

Acute kidney injury is a frequent and serious complication during sepsis. Renal dysfunction occurs through complex mechanisms that involve upregulation of proinflammatory chemokines [85-87], enhanced immune response [87, 88], oxidative stress $[86,88,89]$, increased renal vascular resistance [88], decreased mean arterial pressure [86, 88], loss of hemostasis toward coagulation [90], reduced capillary flow [85, 86, 88], hypoxia [85], and apoptosis [87-89], among several others factors. In that complex scenario, renal vascular permeability likely reflects the physiological condition of ECs in the kidney.

While overexpression of TLR4/NOX-2/ROS/NF- $\mathrm{BB}$ in ECs under endotoxin stimulation has been demonstrated previously [12, 91, 92], its participation in functional endothelial permeability assays has remained elusive until now. Our results showed that a signaling pathway involving TLR4/NOX-2/ROS/NF-kB leads to monolayer compromise, potentially serving as the basis for renal 

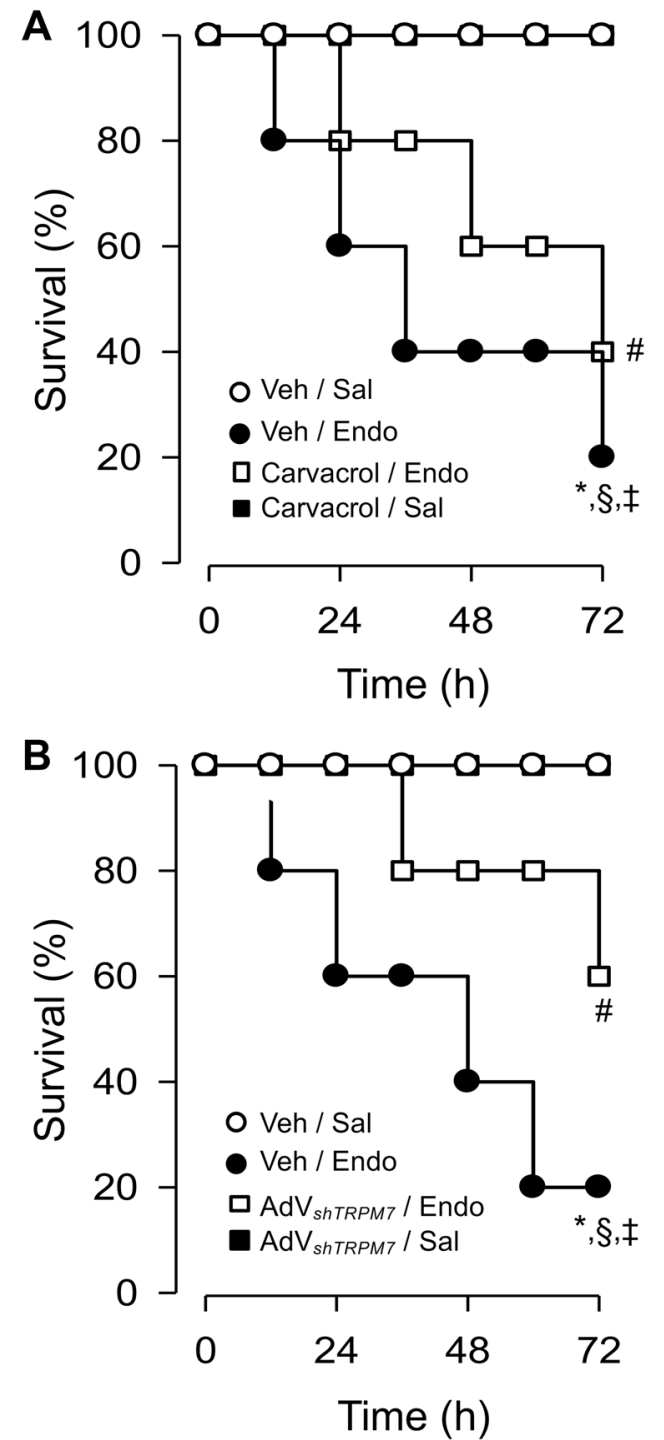

Fig. 6 Survival against endotoxemia is increased by carvacrol treatment and $A_{d V} V_{\text {shTRPM7 }}$ administration. Kaplan-Meier curves depicting $72 \mathrm{~h}$ survival after i.p. administration of $20 \mathrm{mg} / \mathrm{kg}$ LPS or saline solution to animals treated with carvacrol (a), $\operatorname{AdV}_{\text {shTRPM7 }}(\mathbf{b})$, or corresponding vehicle. Statistical differences were assessed by twoway analysis of variance (ANOVA) followed by Bonferroni's post hoc test. ${ }^{*, \$,} p<0.05$, against Veh/Sal condition. $(N=6) .{ }^{\#} p<0.05$, against $\mathrm{Veh} / \mathrm{Sal}$ condition. $(N=10$ per group)

vascular permeability leading to sepsis-induced kidney injury. Recent evidence has shown that the protein expression levels of TLR4 and NF- $\mathrm{KB}$ in kidneys are increased [87, 89]. Moreover, studies have also reported increased protein expression levels of IL-6, IL-1 $\beta$, TNF- $\alpha$, CCL2, and IL-10 in kidneys [87], the presence of inflammatory infiltration [87], and increased markers of oxidative stress [89], all of which cooperate in the pathogenesis of kidney injury during sepsis.

Serial use of inhibiting drugs/elements enables the identification of potential ion channels involved in endotoxin-induced $\mathrm{Ca}^{2+}$ overload and endothelial hyperpermeability. While $\mathrm{Zn}^{2+}, \mathrm{Gd}^{3+}$, and 2-APB, have the broadest impact on ion channels (from voltage-gated to a myriad of other TRP members [93], carvacrol, exerts its effect only on TRPV3 and TRPM7. Carvacrol activates TRPV3 [94] and inhibits TRPM7 [68-70] at concentrations of $\sim 500 \mu \mathrm{M}$. Carvacrol-treated ECs failed to increase $\left[\mathrm{Ca}^{2+}\right]_{\mathrm{i}}$ after endotoxin stimulation. Therefore, it is unlikely that carvacrol stimulates $\mathrm{Ca}^{2+}$ overload via TRPV3 activation. Conversely, carvacrol significantly attenuated the increase in $\left[\mathrm{Ca}^{2+}\right]_{i}$ in ECs challenged with the endotoxin. Therefore, it is likely that carvacrol exerts its effect through the inhibition of TRPM7.

A recent and comprehensive study demonstrated that TRPC6 knockout prevented lung endothelial hyperpermeability ex vivo and in vivo, and even reduced mortality up to $70 \%$ after endotoxin challenge [95]. While pointing to a different TRP channel, these observations may be complementary with our results. Considering that neither TRPC6 nor TRPM7 suppression was sufficient to abolish endotoxin lethality, it seems plausible that different TRP members regulate vascular permeability in an organ/tissue-specific manner. Furthermore, it was reported that salvianolic acid B-induced TRPM6 and TRPM7 decrease showed lessened acute lung injury in endotoxemic rats [96], suggesting that several TRP members could participate regulating organ failure in lungs. Further experiments are required to elucidate this hypothesis. Remarkably, a thorough study using macrophages from endotoxin-challenged TRPM7-deficient mice described that TRPM7 participates in TLR4/NF- $\kappa B$ signaling, mediates intracellular $\mathrm{Ca}^{2+}$ overload, and enables the expression of inflammatory cytokines [49]. This is congruent with another study using an in vivo model of ischemia/reperfusion-related injury, in which it was shown that TRPM7 silencing decreased serum markers of inflammation, apoptosis, tubulointerstitial damage, and renal dysfunction [54]. These observations are consistent with our results and iteratively suggestive of the strong participation of TRPM7 in mediating kidney injury during an inflammatory systemic insult. Nevertheless, presence and activity of TRPM7 is likely not the only reason for endothelial damage and/or organ dysfunction, and may constitute only a part of the constellation of factors contributing to the development of kidney injury and systemic responses during sepsis. A compromise of the endothelial barrier during sepsis has been further evidenced by increased EBD-albumin extravasation and accumulation in the kidney estroma [85-88, 97, 98] and systemically by increased levels of BUN and CRE [8587], reduced clearance of inulin [88], and the presence of albuminuria [97, 99, 100]. Particularly, renal vascular permeability may be enhanced by EC glycocalyx 
degradation. In fact, renal corpuscle damage, reduced syndecan-1 and HA content in renal corpuscles, reduced expression of glomerular sialic acids [101], and increased levels of circulating essential glycocalyx components have been observed during sepsis [102]. Since the EC glycocalyx acts by limiting leukocyte adhesion [103], exacerbated corpuscle inflammatory infiltrates [87] and the inflammatory microenvironment [89] are clear components that deteriorate barrier function. This is consistent with our findings regarding the inflammatory consequences of endotoxemia. After $24 \mathrm{~h}$ of endotoxemia, there is a significant rise in circulating levels of proinflammatory cytokines (Supplementary Table S3) accompanied by morphological changes in the renal cortex (Supplementary Fig. S10) likely contributing to kidney loss-of-function at a physiological level. Moreover, microscopic inspection of the renal cortex from normal and endotoxemic rats showed no significant differences in leukocyte infiltrates in glomeruli or the tubule system (Supplementary Fig. S10). Therefore, both AdV $\mathrm{AnTRPM}_{\text {sh }}$ and carvacrol showed capable of preventing biochemical and morphological features of inflammation $24 \mathrm{~h}$ after endotoxemia was induced, further highlighting the important role of TRPM7 during endotoxemia, especially in the kidney.

Overall, our results showed that under endotoxin stimulation, TRPM7 mediates EC Ca ${ }^{2+}$ overload, TLR4/NOX-2/ ROS/NF- $\mathrm{KB}$ activation, VE-cadherin downregulation, and vascular monolayer disruption. In vivo, under endotoxemia, TRPM7 mediates renal vascular hyperpermeability, kidney dysfunction, and enhanced organism mortality. Therefore, TRPM7 is a remarkable molecular target for the regulation of renal vascular hyperpermeability during endotoxemia and sepsis, making pharmacological and clinical investigation of this target a potentially productive effort to benefit critically ill patients.

Acknowledgements This work was supported by research grants from Fondo Nacional de Desarrollo Científico y Tecnológico (FONDECYT) 1161288, 1160900, and 1161646. Comisión Nacional de Investigación Científica y Tecnológica (CONICYT) Ph.D. Scholarships 21171566 and 21181550. Millennium Institute on Immunology and Immunotherapy P09-016-F. The Millennium Nucleus of Ion Channels-Associated Diseases is a Millennium Nucleus supported by the Iniciativa Científica Milenio of the Ministry of Economy, Development and Tourism (Chile). The authors acknowledge the services provided by UC CINBIOT Animal Facility funded by Program for Associative Research, of the Chilean National Council for Science and Technology (PIA CONICYT) ECM-07.

\section{Compliance with ethical standards}

Conflict of interest The authors declare that they have no conflict of interest.
Publisher's note: Springer Nature remains neutral with regard to jurisdictional claims in published maps and institutional affiliations.

\section{References}

1. Seymour CW, Liu VX, Iwashyna TJ, Brunkhorst FM, Rea TD, Scherag A, et al. Assessment of clinical criteria for sepsis: for the third international consensus definitions for sepsis and septic shock (Sepsis-3). JAMA. 2016;315:762-74.

2. Singer M, Deutschman CS, Seymour CW, Shankar-Hari M, Annane D, Bauer $\mathrm{M}$, et al. The third international consensus definitions for sepsis and septic shock (Sepsis-3). JAMA. 2016;315:801-10.

3. Zarbock A, Gomez H, Kellum JA. Sepsis-induced acute kidney injury revisited: pathophysiology, prevention and future therapies. Curr Opin Crit Care. 2014;20:588-95.

4. Schortgen F, Asfar P. Update in sepsis and acute kidney injury 2014. Am J Respir Crit Care Med. 2015;191:1226-31.

5. Paoli CJ, Reynolds MA, Sinha M, Gitlin M, Crouser E. Epidemiology and costs of sepsis in the United States-an analysis based on timing of diagnosis and severity level. Crit Care Med. 2018;46:1889-97.

6. Schrier RW, Wang W. Acute renal failure and sepsis. N Engl J Med. 2004;351:159-69.

7. Rivers EP, Ahrens T. Improving outcomes for severe sepsis and septic shock: tools for early identification of at-risk patients and treatment protocol implementation. Crit Care Clin. 2008;24: S1-47.

8. Winters BD, Eberlein M, Leung J, Needham DM, Pronovost PJ, Sevransky JE. Long-term mortality and quality of life in sepsis: a systematic review. Crit Care Med. 2010;38:1276-83.

9. Karima R, Matsumoto S, Higashi H, Matsushima K. The molecular pathogenesis of endotoxic shock and organ failure. Mol Med Today. 1999;5:123-32.

10. Riedemann NC, Guo R-F, Ward PA. The enigma of sepsis. J Clin Invest. 2003;112:460-7.

11. Pinsky MR. Dysregulation of the immune response in severe sepsis. Am J Med Sci. 2004;328:220-9.

12. Simon F, Fernández R. Early lipopolysaccharide-induced reactive oxygen species production evokes necrotic cell death in human umbilical vein endothelial cells. $\mathrm{J}$ Hypertens. 2009;27:1202-16.

13. Becerra A, Echeverría C, Varela D, Sarmiento D, Armisén R, Nuñez-Villena F, et al. Transient receptor potential melastatin 4 inhibition prevents lipopolysaccharide-induced endothelial cell death. Cardiovasc Res. 2011;91:677-84.

14. Peters K, Unger RE, Brunner J, Kirkpatrick CJ. Molecular basis of endothelial dysfunction in sepsis. Cardiovasc Res. 2003;60:49-57.

15. Grandel U, Grimminger F. Endothelial responses to bacterial toxins in sepsis. Crit Rev Immunol. 2003;23:267-99.

16. Huet O, Dupic L, Harrois A, Duranteau J. Oxidative stress and endothelial dysfunction during sepsis. Front Biosci. 2011;16:1986-95.

17. Echeverría C, Montorfano I, Sarmiento D, Becerra A, NuñezVillena F, Figueroa XF, et al. Lipopolysaccharide induces a fibrotic-like phenotype in endothelial cells. J Cell Mol Med. 2013;17:800-14.

18. Goldenberg NM, Steinberg BE, Slutsky AS, Lee WL. Broken barriers: a new take on sepsis pathogenesis. Sci Transl Med. 2011;3:88ps25-88ps25.

19. De Backer D, Creteur J, Preiser JC, Dubois MJ, Vincent JL. Microvascular blood flow is altered in patients with sepsis. Am J Respir Crit Care Med. 2002;166:98-104. 
20. Sakr Y, Dubois MJ, De Backer D, Creteur J, Vincent JL. Persistent microcirculatory alterations are associated with organ failure and death in patients with septic shock. Crit Care Med. 2004;32:1825-31.

21. Trzeciak S, Dellinger RP, Parrillo JE, Guglielmi M, Bajaj J, Abate NL, et al. Early microcirculatory perfusion derangements in patients with severe sepsis and septic shock: relationship to hemodynamics, oxygen transport, and survival. Ann Emerg Med. 2007;49:88-98.

22. Dellinger RP, Levy MM, Rhodes A, Annane D, Gerlach H, Opal SM, et al. Surviving sepsis campaign: international guidelines for management of severe sepsis and septic shock, 2012. Intensive Care Med. 2013;39:165-228.

23. Shum H-P, Yan W-W, Chan TM. Recent knowledge on the pathophysiology of septic acute kidney injury: a narrative review. J Crit Care. 2016;31:82-89.

24. Dellinger RP, Levy MM, Rhodes A, Annane D, Gerlach H, Opal SM, et al. Surviving sepsis campaign: international guidelines for management of severe sepsis and septic shock: 2012. Crit Care Med. 2013;41:580-637.

25. Levy MM, Evans LE, Rhodes A. The surviving sepsis campaign bundle: 2018 update. Intensive Care Med. 2018;44:925-8.

26. Levy MM, Evans LE, Rhodes A. The surviving sepsis campaign bundle: 2018 update. Crit Care Med. 2018;46:997-1000.

27. Echeverría C, Montorfano I, Tapia P, Riedel C, CabelloVerrugio C, Simon F. Endotoxin-induced endothelial fibrosis is dependent on expression of transforming growth factors $\beta 1$ and B2. Infect Immun. 2014;82:3678-86.

28. Zeisberg EM, Potenta S, Xie L, Zeisberg M, Kalluri R. Discovery of endothelial to mesenchymal transition as a source for carcinoma-associated fibroblasts. Cancer Res. 2007; 67:10123-8

29. Maleszewska M, Moonen J-RAJ, Huijkman N, et al. IL-1 $\beta$ and TGF 32 synergistically induce endothelial to mesenchymal transition in an NFKB-dependent manner. Immunobiology. 2013; 218:443-54.

30. Mahler GJ, Farrar EJ, Butcher JT. Inflammatory cytokines promote mesenchymal transformation in embryonic and adult valve endothelial cells. Arterioscler Thromb Vasc Biol. 2012;33:121-30.

31. Montorfano I, Becerra A, Cerro R, Echeverría C, Sáez E, Morales MG, et al. Oxidative stress mediates the conversion of endothelial cells into myofibroblasts via a TGF- $\beta 1$ and TGF- $\beta 2$ dependent pathway. Lab Invest. 2014;94:1068-82.

32. Sarmiento D, Montorfano I, Cáceres M, Echeverría C, Fernández $\mathrm{R}$, Cabello-Verrugio $\mathrm{C}$, et al. Endotoxin-induced vascular endothelial cell migration is dependent on TLR4/NF- $\mathrm{KB}$ pathway, $\mathrm{NAD}(\mathrm{P}) \mathrm{H}$ oxidase activation, and transient receptor potential melastatin 7 calcium channel activity. Int $\mathrm{J}$ Biochem Cell Biol. 2014;55:11-23.

33. Echeverría C, Montorfano I, Hermosilla T, Armisén R, Velásquez LA, Cabello-Verrugio C, et al. Endotoxin induces fibrosis in vascular endothelial cells through a mechanism dependent on transient receptor protein melastatin 7 activity. PLoS ONE. 2014;9:e94146.

34. Sutton TA, Kelly KJ, Mang HE, Plotkin Z, Sandoval RM, Dagher PC. Minocycline reduces renal microvascular leakage in a rat model of ischemic renal injury. Am J Physiol Renal Physiol. 2005;288:F91-7.

35. Sutton TA, Mang HE, Campos SB, Sandoval RM, Yoder MC, Molitoris BA. Injury of the renal microvascular endothelium alters barrier function after ischemia. Am J Physiol Renal Physiol. 2003;285:F191-8.

36. Basile DP. The endothelial cell in ischemic acute kidney injury: implications for acute and chronic function. Kidney Int. 2007;72: $151-6$.
37. Chistiakov DA, Orekhov AN, Bobryshev YV. Endothelial PECAM-1 and its function in vascular physiology and atherogenic pathology. Exp Mol Pathol. 2016;100:409-15.

38. Sarelius IH, Glading AJ. Control of vascular permeability by adhesion molecules. Tissue Barriers. 2015;3:e985954.

39. Tiruppathi C, Minshall RD, Paria BC, Vogel SM, Malik AB. Role of $\mathrm{Ca} 2+$ signaling in the regulation of endothelial permeability. Vasc Pharmacol. 2002;39:173-85.

40. Hempel A, Lindschau C, Maasch C, Mahn M, Bychkov R, Noll $\mathrm{T}$, et al. Calcium antagonists ameliorate ischemia-induced endothelial cell permeability by inhibiting protein kinase C. Circulation. 1999;99:2523-9.

41. Townsley MI, King JA, Alvarez DF. Ca2 + channels and pulmonary endothelial permeability: insights from study of intact lung and chronic pulmonary hypertension. Microcirculation. 2006;13:725-39.

42. Shivakumar K, Kumaran C. L-type calcium channel blockers and EGTA enhance superoxide production in cardiac fibroblasts. J Mol Cell Cardiol. 2001;33:373-7.

43. Fonfria E, Murdock PR, Cusdin FS, Benham CD, Kelsell RE, McNulty S. Tissue distribution profiles of the human TRPM cation channel family. J Recept Signal Transduct Res. 2006;26:159-78.

44. Nadler MJ, Hermosura MC, Inabe K, Perraud AL, Zhu Q, Stokes AJ, et al. LTRPC7 is a Mg.ATP-regulated divalent cation channel required for cell viability. Nature. 2001;411:590-5.

45. Simon F, Varela D, Cabello-Verrugio C. Oxidative stressmodulated TRPM ion channels in cell dysfunction and pathological conditions in humans. Cell Signal. 2013;25:1614-24.

46. Clapham DE, Runnels LW, Strübing C. The TRP ion channel family. Nat Rev Neurosci. 2001;2:387-96.

47. Zou Z-G, Rios FJ, Montezano AC, Touyz RM. TRPM7, magnesium, and signaling. Int J Mol Sci. 2019;20:1877.

48. Chubanov V, Mittermeier L, Gudermann T. Role of kinasecoupled TRP channels in mineral homeostasis. Pharmacol Ther. 2018;184:159-76.

49. Schappe MS, Szteyn K, Stremska ME, Mendu SK, Downs TK, Seegren PV, et al. Chanzyme TRPM7 mediates the Ca2+ influx essential for lipopolysaccharide-induced toll-like receptor 4 endocytosis and macrophage activation. Immunity. 2018;48: 59-74.e5.

50. Meng Z, Wang X, Yang Z, Xiang F. Expression of transient receptor potential melastatin 7 up-regulated in the early stage of renal ischemia-reperfusion. Transplant Proc. 2012;44:1206-10.

51. Dusmez D, Cengiz B, Yumrutas O, Demir T, Oztuzcu S, Demiryurek S, et al. Effect of verapamil and lidocaine on TRPM and NaV1.9 gene expressions in renal ischemia-reperfusion. Transplant Proc. 2014;46:33-39.

52. Dokuyucu R, Gogebakan B, Yumrutas O, Bozgeyik I, Gokce H, Demir T. Expressions of TRPM6 and TRPM7 and histopathological evaluation of tissues in ischemia reperfusion performed rats. Ren Fail. 2014;36:932-6.

53. Meng Z, Cao R, Wang Y, Cao H, Liu T, Yang Z, et al. Suppression of renal TRPM7 may alleviate kidney injury in the renal transplantation. World J Urol. 2014;32:1303-11.

54. Liu A, Wu J, Yang C, Wu Y, Zhang Y, Zhao F, et al. TRPM7 in CHBP-induced renoprotection upon ischemia reperfusion-related injury. Sci Rep. 2018;8:5510.

55. Moreno C, Hermosilla T, Morales D, Encina M, Torres-Díaz L, Díaz $P$, et al. Cav $\beta 2$ transcription start site variants modulate calcium handling in newborn rat cardiomyocytes. Pflugers Arch. 2015;467:2473-84.

56. Wang H-L, Lai TW. Optimization of Evans blue quantitation in limited rat tissue samples. Sci Rep. 2014;4:6588.

57. Radu M, Chernoff J. An in vivo assay to test blood vessel permeability. J Vis Exp. 2013;73:e50062-e50062. 
58. Hamer PW, McGeachie JM, Davies MJ, Grounds MD. Evans Blue Dye as an in vivo marker of myofibre damage: optimising parameters for detecting initial myofibre membrane permeability. J Anat. 2002;200:69-79.

59. Archer SL, Cowan NJ. Measurement of endothelial cytosolic calcium concentration and nitric oxide production reveals discrete mechanisms of endothelium-dependent pulmonary vasodilatation. Circ Res. 1991;68:1569-81.

60. Williams DA, Fay FS. Intracellular calibration of the fluorescent calcium indicator Fura-2. Cell Calcium. 1990;11:75-83.

61. Schaff UY, Yamayoshi I, Tse T, Griffin D, Kibathi L, Simon SI. Calcium flux in neutrophils synchronizes $\beta 2$ integrin adhesive and signaling events that guide inflammatory recruitment. Ann Biomed Eng. 2008;36:632-46.

62. Kawamoto T, Ii M, Kitazaki T, lizawa Y, Kimura H. TAK-242 selectively suppresses toll-like receptor 4 -signaling mediated by the intracellular domain. Eur J Pharmacol. 2008;584:40-48.

63. Ii M, Matsunaga N, Hazeki K, Nakamura K, Takashima K, Seya $\mathrm{T}$, et al. A novel cyclohexene derivative, ethyl (6R)-6-[N-(2Chloro-4-fluorophenyl)sulfamoyl]cyclohex-1-ene-1-carboxylate (TAK-242), selectively inhibits toll-like receptor 4-mediated cytokine production through suppression of intracellular signaling. Mol Pharmacol. 2006;69:1288-95.

64. Ni JQ, Ouyang Q, Lin L, Huang Z, Lu H, Chen X, et al. Role of toll-like receptor 4 on lupus lung injury and atherosclerosis in LPS-challenge $\mathrm{ApoE}^{-1-}$ mice. Clin Dev Immunol. 2013;2013: 476856-12.

65. Simon F, Stutzin A. Protein kinase C-mediated phosphorylation of $\mathrm{p} 47 \mathrm{phox}$ modulates platelet-derived growth factor-induced $\mathrm{H}_{2} \mathrm{O}_{2}$ generation and cell proliferation in human umbilical vein endothelial cells. Endothelium. 2008;15:175-88.

66. Hwang J, Kleinhenz DJ, Lassègue B, Griendling KK, Dikalov S, Hart CM. Peroxisome proliferator-activated receptor-gamma ligands regulate endothelial membrane superoxide production. Am J Physiol Cell Physiol. 2005;288:C899-905.

67. Lu Y-C, Yeh W-C, Ohashi PS. LPS/TLR4 signal transduction pathway. Cytokine. 2008;42:145-51.

68. Parnas M, Peters M, Dadon D, Lev S, Vertkin I, Slutsky I, et al. Carvacrol is a novel inhibitor of Drosophila TRPL and mammalian TRPM7 channels. Cell Calcium. 2009;45:300-9.

69. Chen W, Xu B, Xiao A, Liu L, Fang X, Liu R, et al. TRPM7 inhibitor carvacrol protects brain from neonatal hypoxicischemic injury. Mol Brain. 2015;8:11.

70. Chen WL, Barszczyk A, Turlova E, Deurloo M, Liu B, Yang $\mathrm{BB}$, et al. Inhibition of TRPM7 by carvacrol suppresses glioblastoma cell proliferation, migration and invasion. Oncotarget. 2015;6:16321-40.

71. Chokshi R, Fruasaha P, Kozak JA. 2-Aminoethyl diphenyl borinate (2-APB) inhibits TRPM7 channels through an intracellular acidification mechanism. Channels. 2012;6:362-9.

72. Hamaguchi Y, Matsubara T, Amano T, Uetani T, Asano H, Iwamoto $\mathrm{T}$, et al. $\mathrm{Na}+$-independent $\mathrm{Mg} 2+$ transport sensitive to 2-aminoethoxydiphenyl borate (2-APB) in vascular smooth muscle cells: involvement of TRPM-like channels. J Cell Mol Med. 2008;12:962-74.

73. Nuñez-Villena F, Becerra A, Echeverría C, Briceño N, Porras O, Armisén R, et al. Increased expression of the transient receptor potential melastatin 7 channel is critically involved in lipopolysaccharide-induced reactive oxygen species-mediated neuronal death. Antioxid Redox Signal. 2011;15:2425-38.

74. Kozak JA, Cahalan MD. MIC channels are inhibited by internal divalent cations but not ATP. Biophys J. 2003;84:922-7.

75. Levy MM, Fink MP, Marshall JC, Abraham E, Angus D, Cook $\mathrm{D}$, et al. $2001 \mathrm{SCCM} / \mathrm{ESICM} / \mathrm{ACCP} / \mathrm{ATS} / \mathrm{SIS}$ international sepsis definitions conference. Intensive Care Med. 2003; 29:530-8.
76. Levy MM, Fink MP, Marshall JC, Abraham E, Angus D, Cook D, et al. 2001 SCCM/ESICM/ACCP/ATS/SIS international sepsis definitions conference. Crit Care Med. 2003;31:1250-6.

77. Jin J, Desai BN, Navarro B, Donovan A, Andrews NC, Clapham DE. Deletion of Trpm7 disrupts embryonic development and thymopoiesis without altering $\mathrm{Mg} 2+$ homeostasis. Science. 2008;322:756-60.

78. Sun HS, Jackson MF, Martin LJ, Jansen K, Teves L, Cui H. Suppression of hippocampal TRPM7 protein prevents delayed neuronal death in brain ischemia. Nat Neurosci. 2009; 12:1300-7.

79. Sledz CA, Holko M, de Veer MJ, Silverman RH, Williams BR. Activation of the interferon system by short-interfering RNAs. Nat Cell Biol. 2003;5:834-9.

80. Bridge AJ, Pebernard S, Ducraux A, Nicoulaz AL, Iggo R. Induction of an interferon response by RNAi vectors in mammalian cells. Nat Genet. 2003;34:263-4.

81. Lee WL, Slutsky AS. Sepsis and endothelial permeability. N Engl J Med. 2010;363:689-91.

82. Spicer A, Calfee CS. Fixing the leak: targeting the vascular endothelium in sepsis. Crit Care. 2012;16:177.

83. Siddall E, Khatri M, Radhakrishnan J. Capillary leak syndrome: etiologies, pathophysiology, and management. Kidney Int. 2017;92:37-46.

84. Xiao F, Wang D, Kong L, Li M, Feng Z, Shuai B, et al. Intermedin protects against sepsis by concurrently re-establishing the endothelial barrier and alleviating inflammatory responses. Nat Commun. 2018;9:2644.

85. Yasuda H, Yuen PS, Hu X, Zhou H, Star RA. Simvastatin improves sepsis-induced mortality and acute kidney injury via renal vascular effects. Kidney Int. 2006;69:1535-42.

86. Seely KA, Holthoff JH, Burns ST, Wang Z, Thakali KM, Gokden $\mathrm{N}$, et al. Hemodynamic changes in the kidney in a pediatric rat model of sepsis-induced acute kidney injury. Am J Physiol Renal Physiol. 2011;301:F209-17.

87. Chen L, Yang S, Zumbrun EE, Guan H, Nagarkatti PS, Nagarkatti M. Resveratrol attenuates lipopolysaccharide-induced acute kidney injury by suppressing inflammation driven by macrophages. Mol Nutr Food Res. 2015;59:853-64.

88. Kim DH, Jung YJ, Lee AS, et al. COMP-angiopoietin-1 decreases lipopolysaccharide-induced acute kidney injury. Kidney Int. 2009;76:1180-91.

89. Kim DH, Jung YJ, Lee AS, Lee S, Kang KP, Lee TH, et al. Icariin improves sepsis-induced mortality and acute kidney injury. Pharmacology. 2018;102:196-205.

90. Melican K, Boekel J, Månsson LE, Sandoval RM, Tanner GA, Källskog $\mathrm{O}$, et al. Bacterial infection-mediated mucosal signalling induces local renal ischaemia as a defence against sepsis. Cell Microbiol. 2008;10:1987-98.

91. Sun R, Zhu Z, Su Q, Li T, Song Q. Toll-like receptor 4 is involved in bacterial endotoxin-induced endothelial cell injury and SOC-mediated calcium regulation. Cell Biol Int. 2012;36:475-81.

92. Wang Y, Zhang MX, Meng X, Liu FQ, Yu GS, Zhang C, et al. Atorvastatin suppresses LPS-induced rapid upregulation of Tolllike receptor 4 and its signaling pathway in endothelial cells. Am J Physiol Heart Circ Physiol. 2011;300:H1743-52.

93. Clapham DE, Julius D, Montell C, Schultz G. International union of pharmacology. XLIX. Nomenclature and structure-function relationships of transient receptor potential channels. Pharmacol Rev. 2005;57:427-50.

94. Xu HX, Delling M, Jun JC, Clapham DE. Oregano, thyme and clove-derived flavors and skin sensitizers activate specific TRP channels. Nat Neurosci. 2006;9:628-35.

95. Tauseef M, Knezevic N, Chava KR, Smith M, Sukriti S, Gianaris $\mathrm{N}$, et al. TLR4 activation of TRPC6-dependent calcium signaling 
mediates endotoxin-induced lung vascular permeability and inflammation. J Exp Med. 2012;209:1953-68.

96. Yang CW, Liu H, Li XD, Sui SG, Liu YF. Salvianolic acid B protects against acute lung injury by decreasing TRPM6 and TRPM7 expressions in a rat model of sepsis. J Cell Biochem. 2018;119:701-11.

97. von Drygalski A, Furlan-Freguia C, Ruf W, Griffin JH, Mosnier LO. Organ-specific protection against lipopolysaccharideinduced vascular leak is dependent on the endothelial protein C receptor. Arterioscler Thromb Vasc Biol. 2013; 33:769-76.

98. Temmesfeld-Wollbrück B, Brell B, Dávid I, Dorenberg M, Adolphs J, Schmeck B, et al. Adrenomedullin reduces vascular hyperpermeability and improves survival in rat septic shock. Intensive Care Med. 2007;33:703-10.

99. De Gaudio AR, Adembri C, Grechi S, Novelli GP. Microalbuminuria as an early index of impairment of glomerular permeability in postoperative septic patients. Intensive Care Med. 2000;26:1364-8.

100. Salmon AHJ, Satchell SC. Endothelial glycocalyx dysfunction in disease: albuminuria and increased microvascular permeability. $\mathbf{J}$ Pathol. 2012;226:562-74.

101. Adembri C, Sgambati E, Vitali L, Selmi V, Margheri M, Tani A, et al. Sepsis induces albuminuria and alterations in the glomerular filtration barrier: a morphofunctional study in the rat. Crit Care. 2011;15:R277.

102. Margraf A, Herter JM, Kühne K, Stadtmann A, Ermert T, Wenk M, et al. 6\% Hydroxyethyl starch (HES 130/0.4) diminishes glycocalyx degradation and decreases vascular permeability during systemic and pulmonary inflammation in mice. Crit Care. 2018;22:111.

103. Lipowsky HH. The endothelial glycocalyx as a barrier to leukocyte adhesion and its mediation by extracellular proteases. Ann Biomed Eng. 2012;40:840-8. 